ARTICLE

\title{
Alpha-ketoglutarate ameliorates age-related osteoporosis via regulating histone methylations
}

\author{
Yuan Wang ${ }^{1,4}$, Peng Deng (10 2,4, Yuting Liu1, Yunshu Wu', Yaqian Chen', Yuchen Guo ${ }^{1}$, Shiwen Zhang ${ }^{1}$, \\ Xiaofei Zheng ${ }^{1,3}$, Liyan Zhou ${ }^{1}$, Weiqing Liu', Qiwen Li ${ }^{1}$, Weimin Lin ${ }^{1}$, Xingying Qi ${ }^{1}$, Guomin Ou${ }^{1}$, Cunyu Wang ${ }^{2}$ \& \\ Quan Yuan (10) 1 (ख
}

\begin{abstract}
Age-related osteoporosis is characterized by the deterioration in bone volume and strength, partly due to the dysfunction of bone marrow mesenchymal stromal/stem cells (MSCs) during aging. Alpha-ketoglutarate $(\alpha K G)$ is an essential intermediate in the tricarboxylic acid (TCA) cycle. Studies have revealed that $\alpha K G$ extends the lifespan of worms and maintains the pluripotency of embryonic stem cells (ESCs). Here, we show that the administration of $\alpha K G$ increases the bone mass of aged mice, attenuates age-related bone loss, and accelerates bone regeneration of aged rodents. $\alpha K G$ ameliorates the senescence-associated (SA) phenotypes of bone marrow MSCs derived from aged mice, as well as promoting their proliferation, colony formation, migration, and osteogenic potential. Mechanistically, aKG decreases the accumulations of H3K9me3 and H3K27me3, and subsequently upregulates BMP signaling and Nanog expression. Collectively, our findings illuminate the role of $\alpha \mathrm{KG}$ in rejuvenating MSCs and ameliorating age-related osteoporosis, with a promising therapeutic potential in age-related diseases.
\end{abstract}

\footnotetext{
${ }^{1}$ State Key Laboratory of Oral Diseases, National Clinical Research Center for Oral Diseases, West China Hospital of Stomatology, Sichuan University, Chengdu, China. ${ }^{2}$ Laboratory of Molecular Signaling, Division of Oral Biology and Medicine, School of Dentistry and Jonsson Comprehensive Cancer Center, UCLA, Los Angeles, CA 90095, USA. ${ }^{3}$ Present address: Department of Stomatology, The First Affiliated Hospital of Zhengzhou University, Zhengzhou, China.

${ }^{4}$ These authors contributed equally: Yuan Wang, Peng Deng. ${ }^{凶}$ email: yuanquan@scu.edu.cn
} 
A ging is characterized by the chronic and gradual deterioration of the functional capacities at the cellular, tissue, and organismal levels, making the individual more prone to various diseases, such as osteoporosis ${ }^{1}$. Osteoporosis is the systemic loss of bone mass and exhibits significant degradation in mechanical properties, which subsequently leads to an increased risk of bone fracture ${ }^{2}$. As the aging population is dramatically rising worldwide, the incidence of osteoporosis is significantly increasing as well, which creates a huge burden on public health ${ }^{3}$.

Recently, several therapies including caloric restriction, telomerase-based therapies, antioxidant supplementation, senolytic therapeutics, cellular reprogramming, and administration of epigenetic regulators (sirtuins activators, histone deacetylases inhibitors, noncoding miRNAs regulators, etc.) have been reported to have overall anti-aging effects, while the efficacy and biocompatibility of those therapies are still dubious ${ }^{4}$. As for the treatment of age-associated osteoporosis, FDA/Europe-approved treatments represented by bisphosphonates ${ }^{5}$ and recombinant human parathyroid hormone exert their therapeutic effects via either anabolic or anti-resorptive function ${ }^{6}$. However, their uses in long-term treatment are limited due to their adverse effects such as osteosarcoma, osteonecrosis of jaw, and atypical femur fractures ${ }^{6,7}$. Although there are various new drugs (Romosozu$\mathrm{mab}^{8}$, Abaloparatide ${ }^{9}$, Cathepsin $\mathrm{K}$ inhibitors ${ }^{10}$, etc.) and novel therapeutic targets (Semaphorin $3 \mathrm{~A}^{11}$, EphrinB2/EphB4 ${ }^{12}$, etc.), lack of efficacy and occurrence of corresponding side effects impede their application in osteoporosis treatment ${ }^{13}$.

Alpha-ketoglutarate $(\alpha K G)$ is a crucial intermediate in the tricarboxylic acid (TCA) cycle, locating between succinyl-CoA and isocitrate. As a key point of anaplerotic reaction, aKG regulates ATP production and reduces equivalent $(\mathrm{NAD}+/ \mathrm{NADH})$ generation in the TCA cycle, therefore influencing ROS level and immune system homeostasis ${ }^{14}$. Moreover, $\alpha \mathrm{KG}$ is an important source of glutamine and glutamate, which are required for the synthesis of both amino acid and collagen ${ }^{15}$.

Administration of either aKG or its derivatives can promote bone development in the growing rats and lambs ${ }^{16,17}$, and protect bone loss induced by hormone deficiency ${ }^{18-20}$. Notably, recent studies reveal a pivotal role of aKG in maintaining the pluripotency of embryonic stem cells (ESCs) ${ }^{21}$ and in anti-aging therapy $^{22}$. Administration of $\alpha \mathrm{KG}$ extends the lifespan and delays the decline of rapid, coordinated body movement of worms, in a dose-dependent manner ${ }^{22}$. However, its influence on the agerelated osteoporosis remains to be investigated.

In this study, we show that supplementation of aKG increases bone mass of aged mice, protects bone loss in adult mice, and accelerates bone regeneration of aged rodents. In vitro treatment with aKG ameliorates the age-related phenotypes of MSCs, along with promoting their proliferation, migration, and osteogenesis. Mechanistically, aKG decreases the accumulations of $\mathrm{H} 3 \mathrm{~K} 9 \mathrm{me} 3$ and $\mathrm{H} 3 \mathrm{~K} 27 \mathrm{me} 3$, and reduces their enrichments on the promoters of Bmp2, Bmp4, and Nanog.

\section{Results}

aKG increases the bone mass of aged mice. To study the potential role of $\alpha \mathrm{KG}$ in age-related osteoporosis, we first measured the circulating levels of aKG in aged rodents (mice, 18months-old; rats, 24-months-old) and found they were significantly lower than those of young adults (3-months-old) (Supplementary Fig. 1a, b). Administration with 0.25 or $0.75 \%$ aKG in drinking water successfully restored the serum levels in aged mice (Fig. 1a, b), without a significant change of body weight (Supplementary Fig. 2a). MicroCT analyses showed that vertebral bone volume (BV/TV) of both female and male animals were elevated after aKG administration (Fig. 1c, d and Supplementary
Fig. 3a, b). Consistently, the trabecular thickness (Tb.Th) and trabecular number (Tb.N) were increased, accompanied with decreased trabecular separation (Tb.Sp) (Fig. 1d and Supplementary Fig. 3b). Analogously, $\mathrm{aKG}$ administration also increased the bone mass of femur and tibia (Supplementary Fig. 4a-d). Analyses of femoral midshaft revealed an increased cortical bone mineral density (BMD) in aKG-treated mice, while cortical thickness (Ct.Th) was relatively even in all groups (Supplementary Fig. 4e, f).

Histomorphometric analyses confirmed the increased bone mass in aKG-treated groups, especially in $0.75 \%$ group (Fig. 1e and Supplementary Fig. 3c). aKG-treated mice displayed an increased number of osteoblasts (N.Ob/B.Pm) and osteoclasts (N.Oc/B.Pm) (Fig. 1f and Supplementary Fig. 3d), indicating a more active bone remodeling status. Higher mineral apposition rate (MAR) and bone formation rate (BFR) were also observed in aKG groups (Fig. If and Supplementary Fig. 3d).

Next, we isolated the MSCs from aged female mice treated with 1 -month $0.75 \%$ aKG or vehicle, and cultured them in osteogenic induction medium. As determined by the alkaline phosphatase (ALP) and Alizarin Red S (ARS) staining, MSCs isolated from the aKG-treated mice exhibited a stronger osteogenic potential than that of the controls (Fig. 1g-i). In addition, the expression of the osteogenic-related genes, such as Runx2, Sp7, Colla1, Alp, and Bglap, was upregulated in MSCs isolated from the aKG-treated mice (Fig. 1j).

aKG attenuates age-related bone loss. The trabecular bone mass of C57BL/6J mice reaches the peak when they become sexually mature (about 2-month-old) and declines sharply in the next few months ${ }^{23,24}$. We next sought to investigate if aKG is capable of attenuating age-related bone loss in adult female mice (Fig. 2a). No significant difference in body weight was observed during the experimental period (Supplementary Fig. 2b). Adult female mice suffered from a $77.5 \%$ reduction of femoral trabecular bone volume from 2-month-old to 6-month-old in the control group (Fig. 2b-d). Interestingly, supplementation of $0.25 \%$ aKG in the drinking water attenuated the trabecular bone loss. $75.3 \%$ and $49.1 \% \mathrm{BV} / \mathrm{TV}$ were maintained at 4-month-old and 6-month-old compared to 2-month-old mice, respectively (Fig. 2d). Consistently, the reduction in Tb.N and the progressive increase in Tb.Sp became moderate in the experimental group than the control group (Fig. 2d). In accordance with our findings in aged mice, the numbers of osteoblasts and osteoclasts were also increased in aKG-treated mice at 4-month and 6-month-old in comparison to the controls (Fig. 2e, f). Likewise, aKG decreased the age-related trabecular bone loss in tibiae and vertebrae (Supplementary Fig. 5a-f).

aKG accelerates bone defect healing of aged rodents. The healing of bone defect is postponed along with aging ${ }^{25}$. Here we assessed the influence of $\alpha \mathrm{KG}$ on bone healing of aged rats using a femoral defect model induced by drilling-hole injury. We started feeding aged rats with $0.75 \%$ aKG from 2 weeks prior to the surgery (Fig. 3a), and successfully elevated the circulating level of aKG (Fig. 3b). Both BMD and BV/TV were increased in the aKG-treated group compared with the controls at 2 weeks and 4 weeks after surgery (Fig. 3c, d). Histomorphometric analyses confirmed the increase of BV/TV, and numbers of osteoblasts and osteoclasts (Fig. 3e-g).

aKG promotes proliferation and migration of aged MSCs. As the aging of MSC is accompanied with deterioration of proliferation capacity, colony-formation ability, migration activity, and osteogenic differentiation 26,27 , we sought to investigate 
a

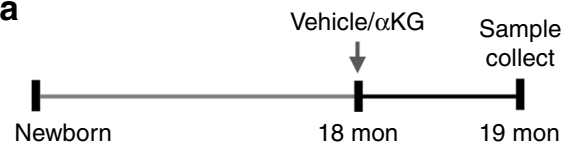

b

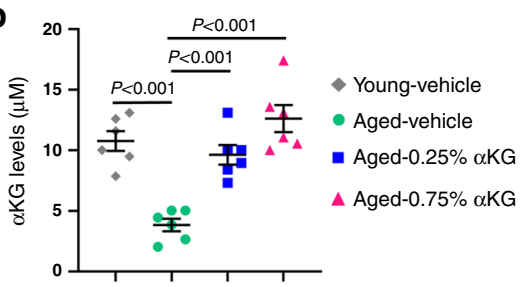

C
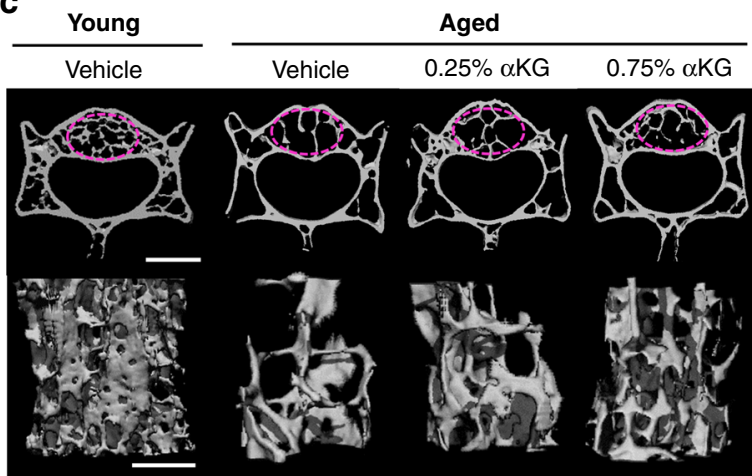

d
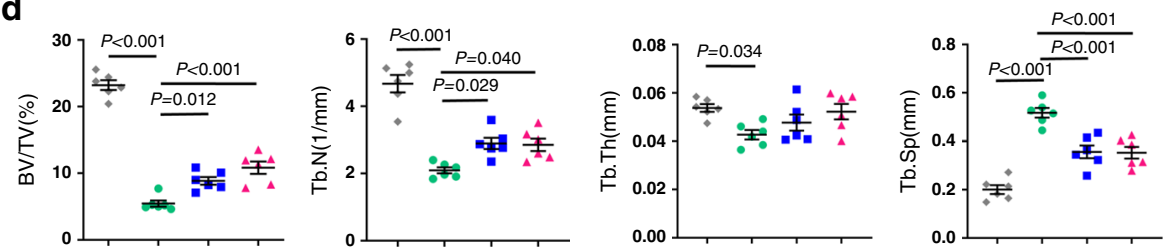

Young-vehicle

- Aged-vehicle

- Aged $-0.25 \% \alpha \mathrm{KG}$

Aged $-0.75 \% \alpha \mathrm{KG}$

e

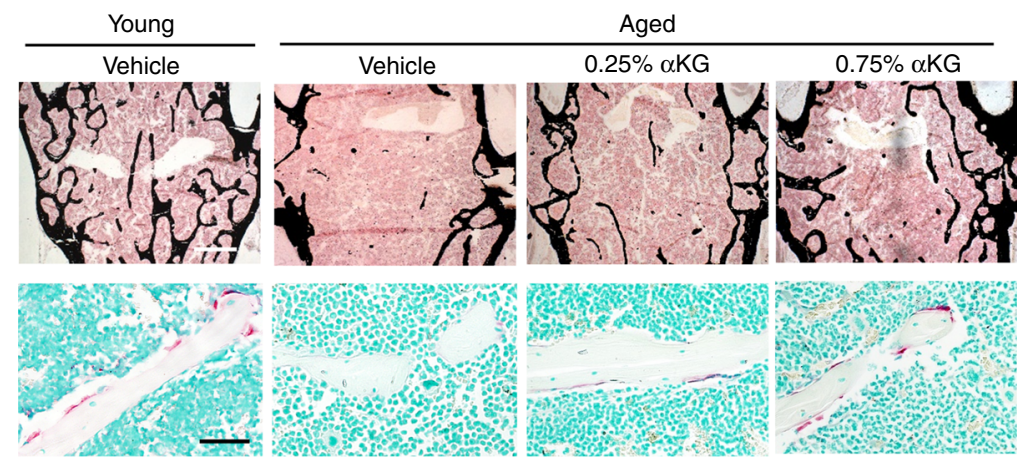

f
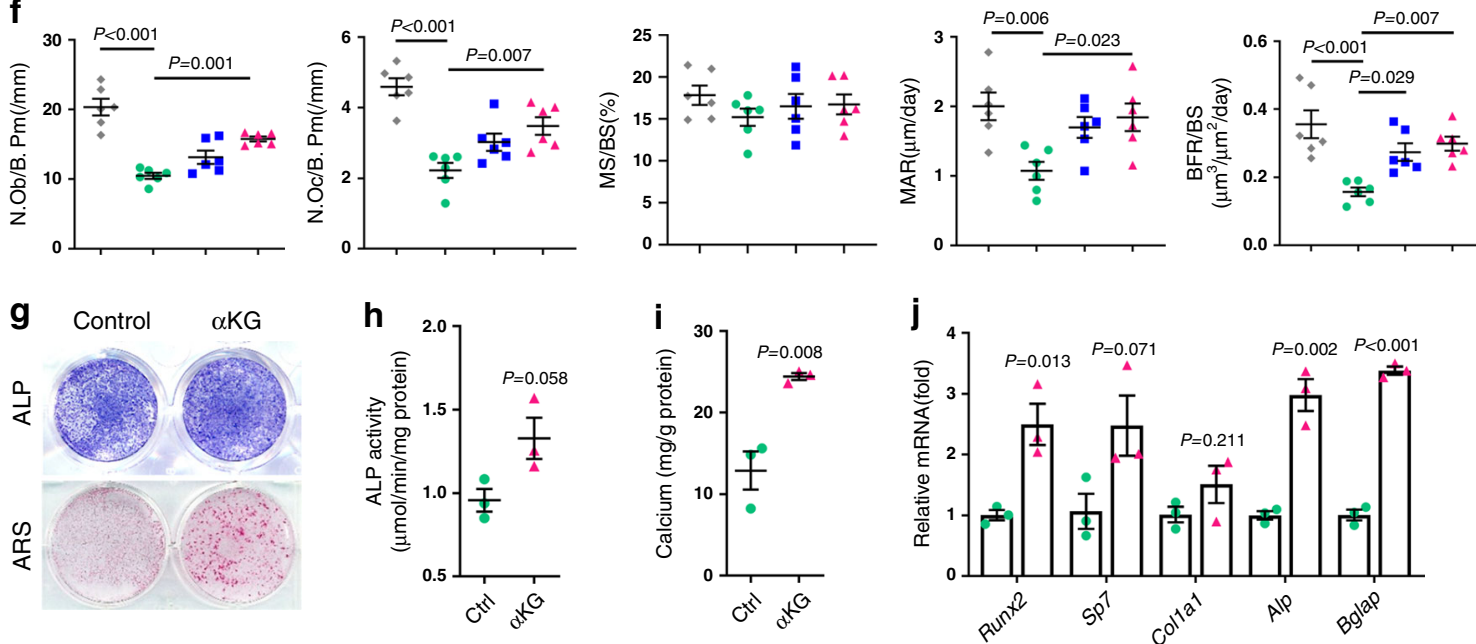

whether aKG had any influence on those biological characteristics of aged MSCs. We observed a drop of intracellular aKG level in MSCs isolated from aged mice in comparison to those from young adults (Fig. 4a). Supplementation of aKG in culture medium successfully elevated the intracellular aKG levels (Fig. 4b) and increased EdU ${ }^{+}$cells without affecting cell apoptosis (Fig. 4c and Supplementary Fig. 6a-c). In addition, aKG improved the colony formation capacity of aged MSCs (Fig. 4d, e). Next, we investigated the migration of MSCs through scratch assay and transwell assay in the presence or absence of $2 \mathrm{mM}$
aKG. At 9 and $18 \mathrm{~h}$ after scratching, the aKG-treated group showed an accelerated speed of wound healing (Fig. 4f, g). Consistently, the migration activity of aged MSCs was also enhanced by aKG treatment in transwell assay (Fig. $4 \mathrm{~h}, \mathrm{i}$ ).

aKG stimulates osteogenic differentiation of aged MSCs. To evaluate the effect of $\alpha \mathrm{KG}$ on osteogenic differentiation of aged MSCs in vitro, we isolated bone marrow MSCs from 18-monthold mice and cultured in osteogenic induction medium. aKG 
Fig. $1 \alpha K G$ increases the bone mass of aged female mice. a Schematic representation of $\alpha K G$ administration. $\mathbf{b} \alpha K G$ administration increased circulating $\alpha K G$ levels of aged mice ( $n=6$, by one-way ANOVA with Tukey's post hoc test). c Representative images of microCT reconstruction of lumber 4 (L4) vertebrae. Scale bar, $1 \mathrm{~mm}$ (upper) or $500 \mu \mathrm{m}$ (lower). The magenta circles indicate the region of interest (ROI). d Quantitative microCT analyses of trabecular bone of L4 vertebrae ( $n=6$, by one-way ANOVA with Tukey's post hoc test). Bone volume (BV/TV, \%); trabecular number (Tb.N, 1/mm); trabecular thickness (Tb.Th, mm); trabecular separation (Tb.Sp, mm). e Representative Von Kossa staining and TRAP staining images of L4 vertebrae. Scale bar, $500 \mu \mathrm{m}$ (upper) or $50 \mu \mathrm{m}$ (lower). $\mathbf{f}$ Histomorphometric analyses of L4 vertebrae $(n=6$, by one-way ANOVA with Tukey's post hoc test). Number of osteoblasts (N.Ob/B.Pm, 1/mm); Number of osteoclasts (N.Oc/B.Pm, 1/mm); Mineral apposition rate (MAR, $\mu \mathrm{m} /$ day); Mineralizing surface per bone surface (MS/BS, \%); Bone formation rate per bone surface (BFR/BS, $\mu \mathrm{m}^{3} / \mu \mathrm{m}^{2} /$ day). $\mathbf{g}$ Representative images of alkaline phosphatase (ALP) and Alizarin Red S (ARS) staining of bone marrow MSCs isolated from the aged mice with or without $\alpha \mathrm{KG}$ treatment. $\mathbf{h}$ Quantitative analysis of the ALP activity ( $n=3$, by two-tailed Student's $t$-test). i Quantitative analysis of the mineralization ( $n=3$, by two-tailed Student's $t$-test). $\mathbf{j}$ Quantitative RT-PCR results of mRNA expression of Runx2, Sp7, Colla1, Alp, and Bglap in MSCs isolated from vehicle/ $\alpha$ KG treated mice ( $n=3$, by two-tailed Student's $t$-test). All data are shown as mean \pm SEM.

a

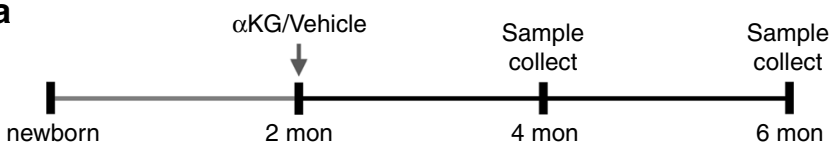

b
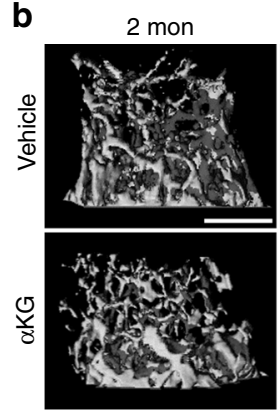

4 mon
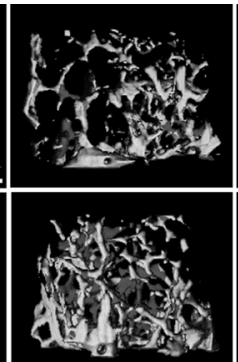

6 mon

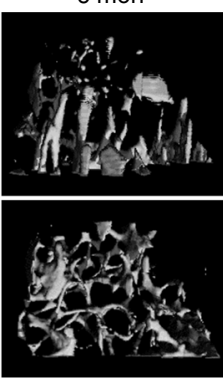

c

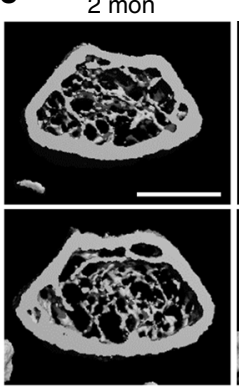

4 mon

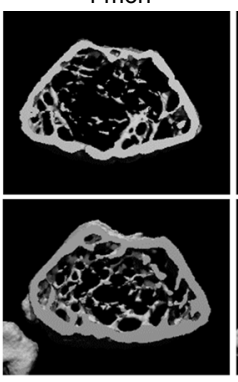

6 mon

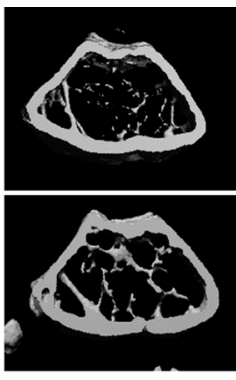

d
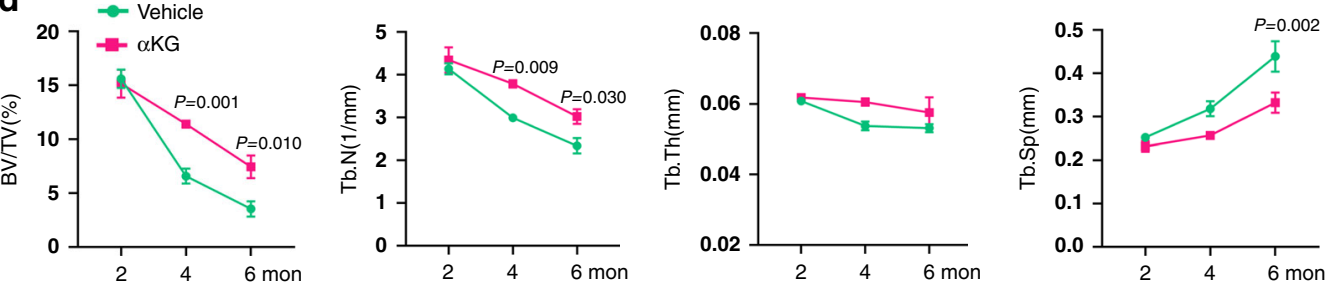

e
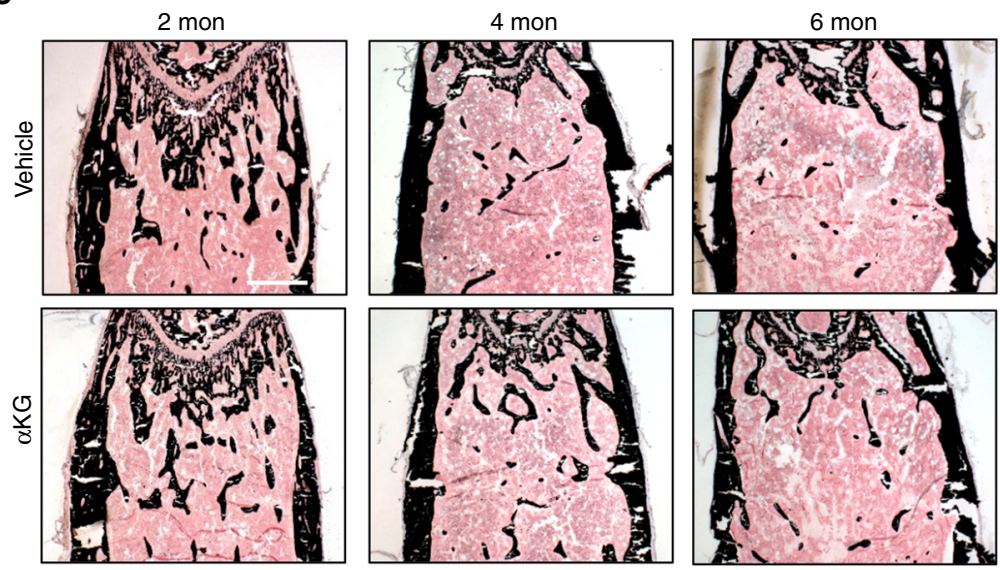

f

f $\rightarrow$ Vehicle $\quad-\alpha K G$
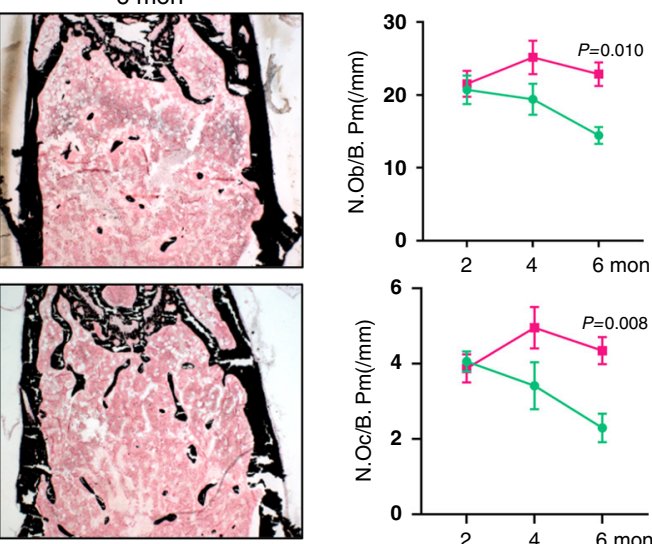

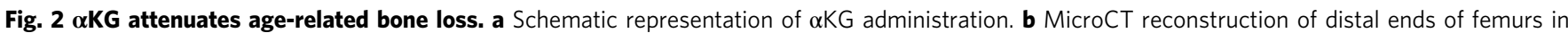
longitudinal direction. Scale bar, $500 \mu \mathrm{m}$. c MicroCT reconstruction of distal ends of femurs in horizontal direction. Scale bar, $1 \mathrm{~mm}$. d Quantitative microCT analyses of distal femurs $(n=6)$. e Von Kossa staining of undecalcified sections of femurs. Scale bar, $500 \mu m$. $\mathbf{f}$ Histomorphometric analyses of distal femurs $(n=6)$. All data are shown as mean \pm SEM. The $P$ values were calculated by two-way ANOVA with Sidak's multiple comparisons test. 
a
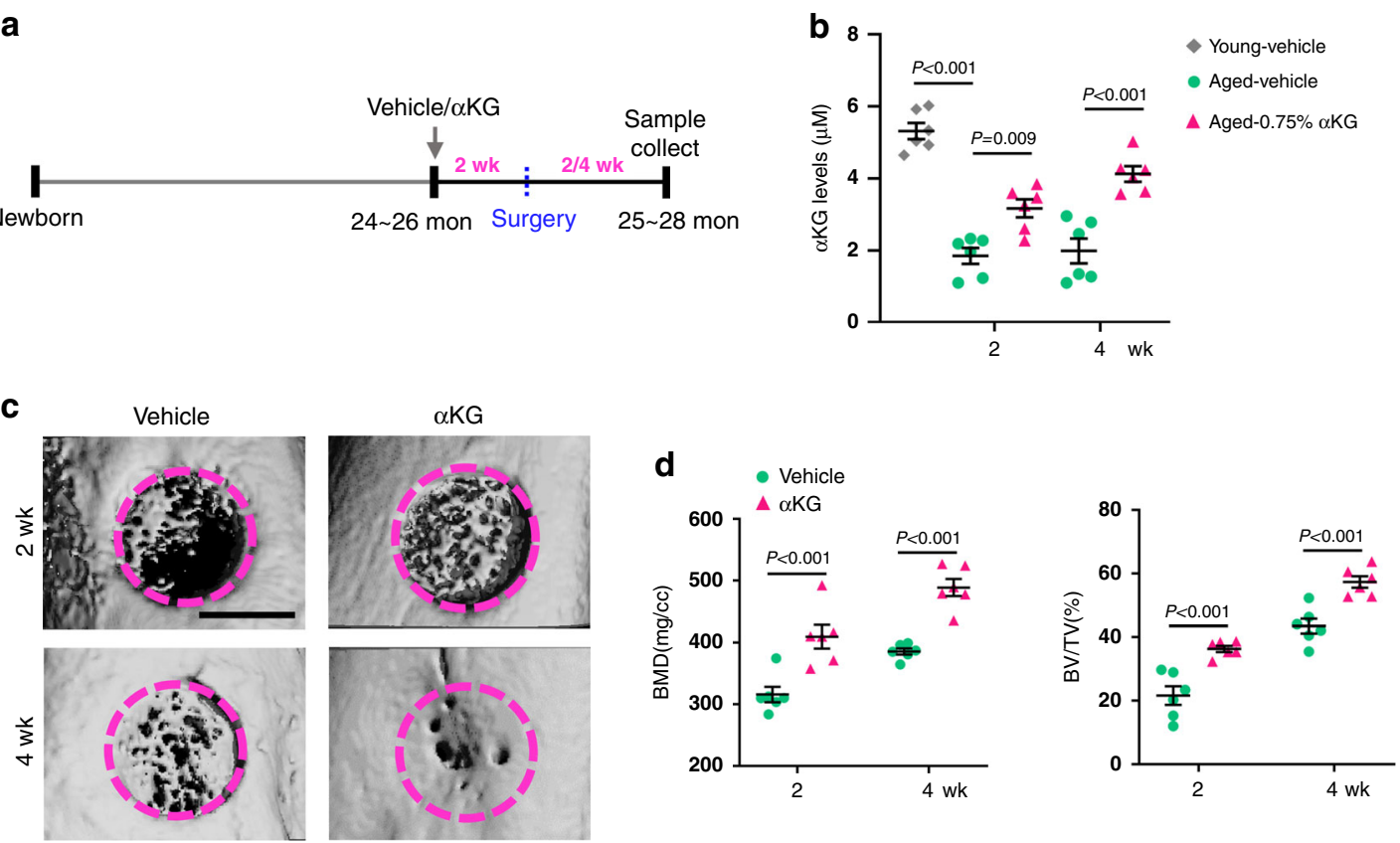

e
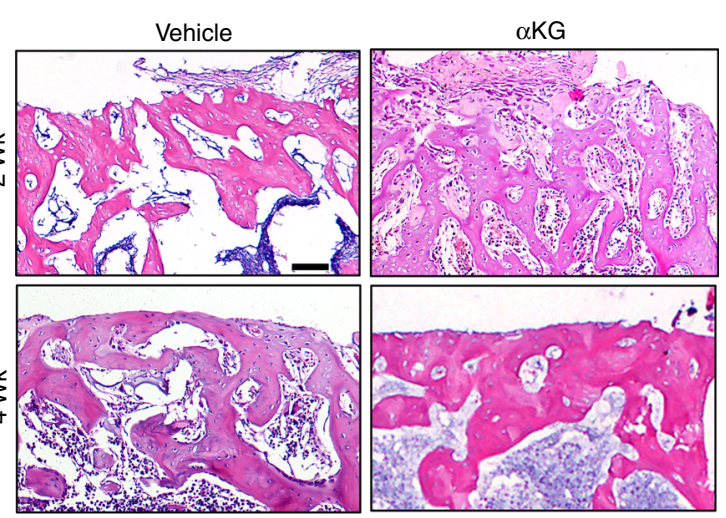

f
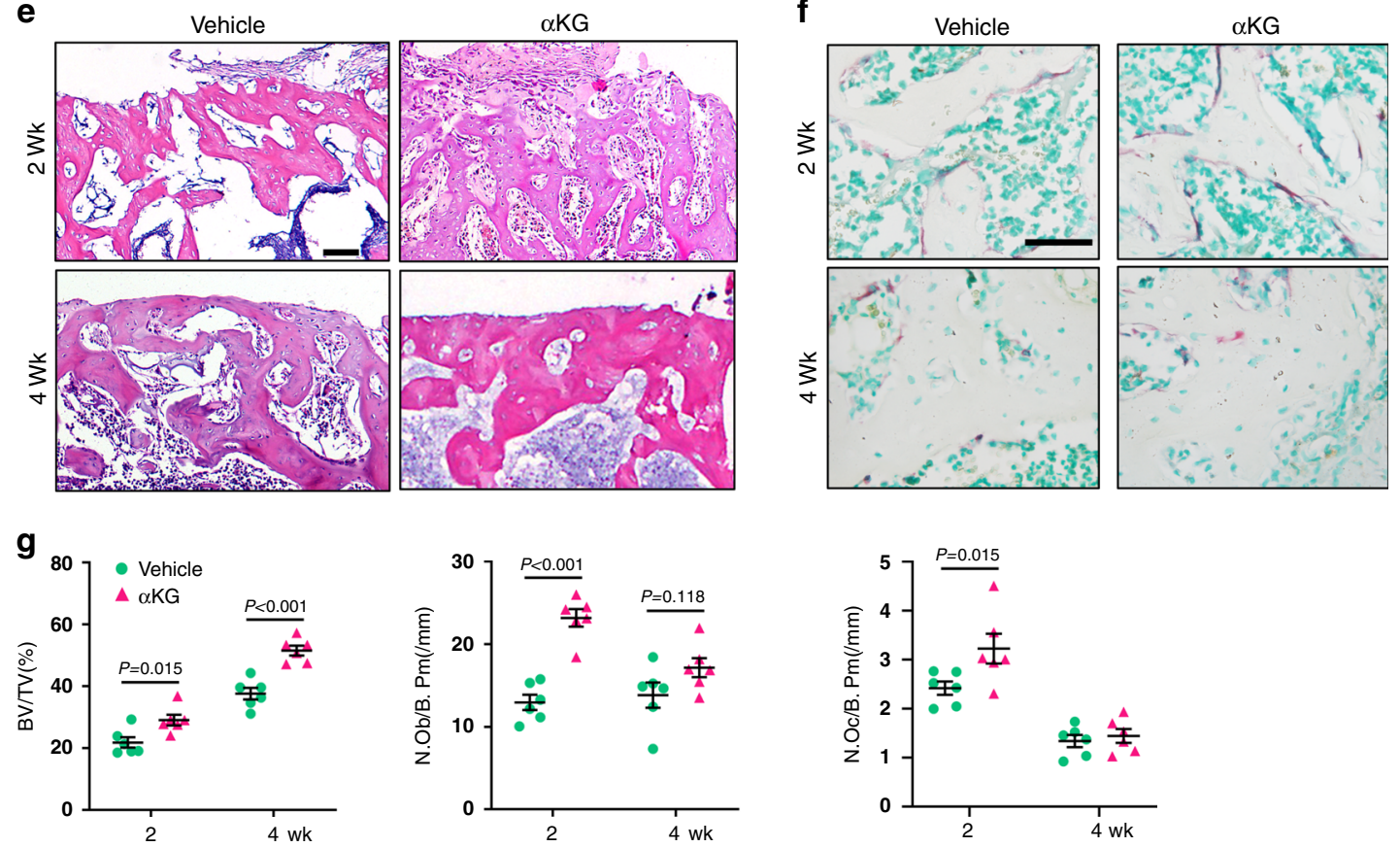

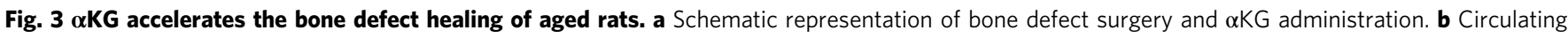
$\alpha K G$ levels after $\alpha K G$ administration ( $n=6$, by one-way ANOVA with Tukey's post hoc test). c Representative images of microCT reconstruction of femoral cortical bone defects at 2 and 4 weeks. The magenta dotted lines indicate the original position of the defect margin. Scale bar, 1 mm. $\mathbf{d}$ Quantitative microCT analyses of bone mineral density (BMD, mg/cc) and bone volume/total volume (BV/TV, \%) within the original defect area $(n=6$, by two-way ANOVA with Sidak's multiple comparisons test). e, $\mathbf{f}$ Representative images of H\&E staining and TRAP staining of femoral cortical bone defects. Scar bar, $100 \mu \mathrm{m}$ (H\&E), $50 \mu \mathrm{m}$ (TRAP). $\mathbf{g}$ Histomorphometric analyses within the bone defect ( $n=6$, by two-way ANOVA with Sidak's multiple comparisons test). Data are expressed as mean \pm SEM.

supplement augmented ALP activity after 7 days of osteogenic induction (Fig. $4 \mathrm{j})$ and increased the formation of mineralized nodules after 3 weeks (Fig. 4j). These findings were confirmed by quantitative analyses of ALP activity and calcium deposition (Fig. 4k, l). Van Gieson staining also showed that $\alpha \mathrm{KG}$ enhanced collagen production (Fig. 4j). Consistently, aKG treatment elevated the expression of the osteogenic-related genes, such as Runx2, Sp7, Colla1, Alp, and Bglap (Fig. 4m).
aKG ameliorates age-associated hallmarks of aged MSCs. To evaluate the effect of aKG on cell senescence, we isolated bone marrow MSCs from 18-month-old mice and treated with $2 \mathrm{mM}$ aKG. The treated cells exhibited a longer lifespan and shorter population-doubling time, compared to the control cells (Fig. 5a). The expression of aging markers, such as $p 16, p 21, p 53$, and $I l 6^{27}$, was significantly downregulated in response to aKG treatment (Fig. 5b). Besides, supplementation of aKG restored the nuclear 
a

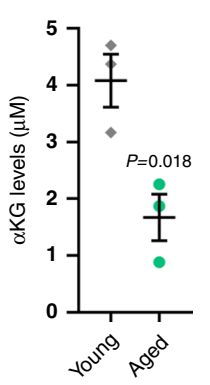

b

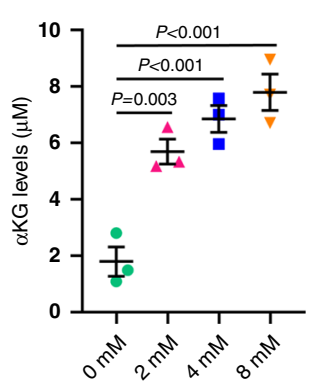

C

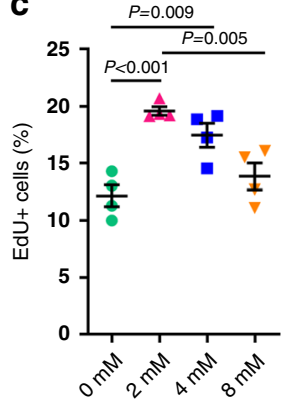

d

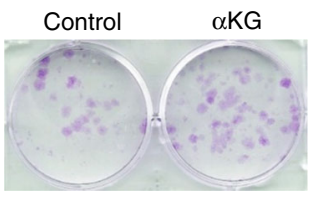

e

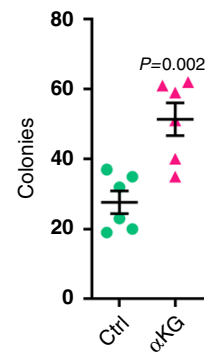

f Control
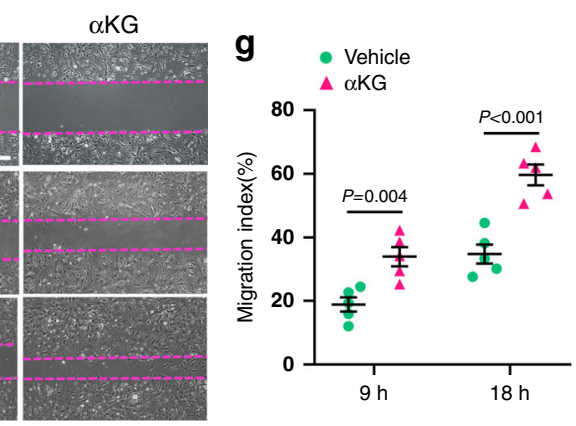

h
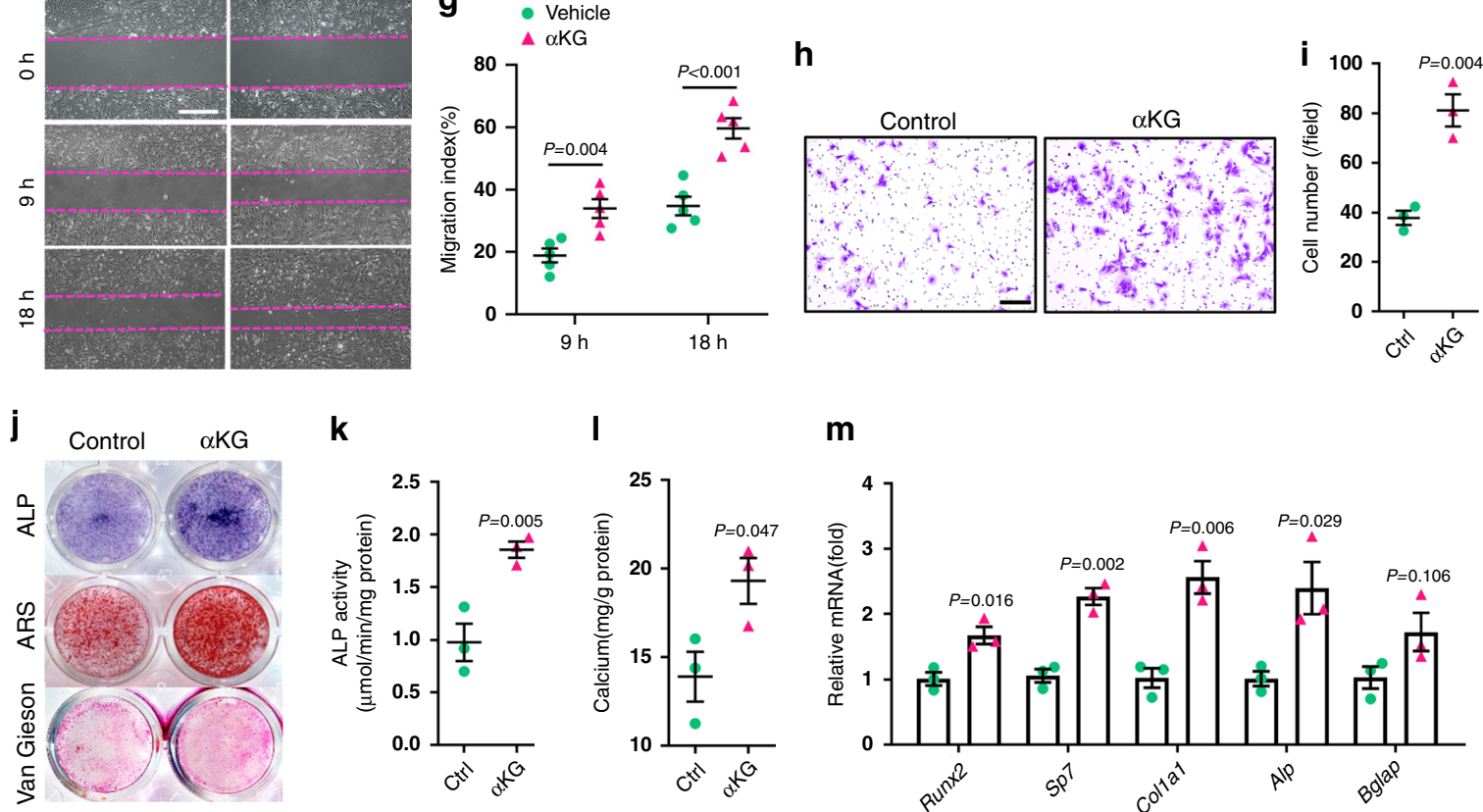

$\mathbf{k}$

I
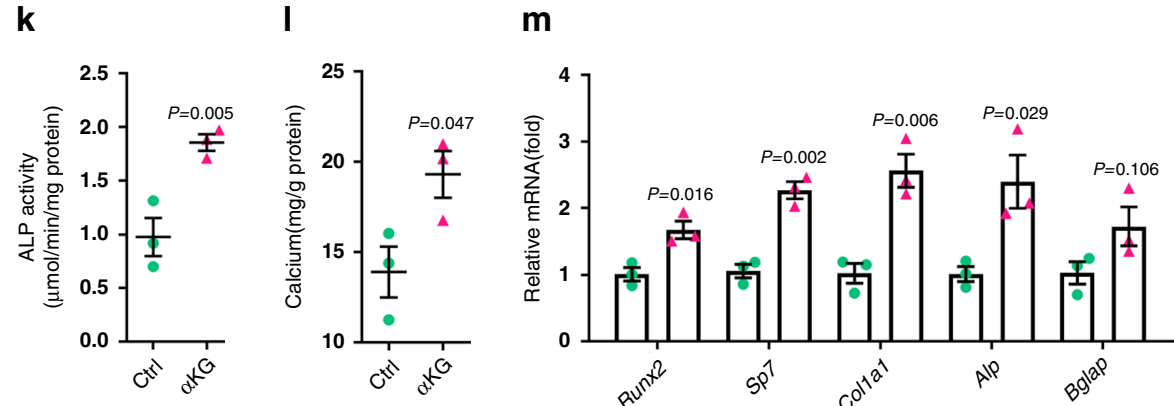

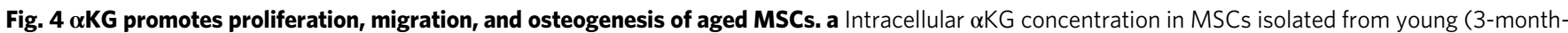
old) and aged (18-month-old) mice ( $n=3$, by two-tailed Student's $t$-test). $\mathbf{b}$ Intracellular $\alpha K G$ concentration in aged MSCs in response to $\alpha K G$ treatment for 3 days ( $n=3$, by one-way ANOVA with Tukey's post hoc test). c EdU incorporation in aged MSCs treated with different concentrations of $\alpha$ KG ( $n=4$, by one-way ANOVA with Tukey's post hoc test). $\alpha$ KG treatment increased the number of EdU + cells. $\mathbf{d}$ Crystal violet staining of colony-forming units of MSCs. e Quantitative analysis of colony-forming unit assay (colonies per well, $n=6$, by two-tailed Student's $t$-test). $\mathbf{f}$, $\mathbf{g}$ Scratch assay of aged MSCs and quantitative analyses. Magenta dotted lines indicate start $(0 \mathrm{~h})$, half $(9 \mathrm{~h})$, and end $(18 \mathrm{~h})$ positions of MSCs after scraping ( $n=5$, by two-way ANOVA with Sidak's multiple comparisons test). Scale bar, $500 \mu \mathrm{m}$. h, i Crystal violet staining of migrated MSCs from the upper chamber to bottom lower of the membrane and quantitative results ( $n=3$, by two-tailed Student's $t$-test). Scale bar, $100 \mu \mathrm{m}$. $\mathbf{j}$ Representative images of ALP, ARS and Van Gieson staining of aged MSCs. k Quantitative analysis of the ALP activity in MSCs ( $n=3$, by two-tailed Student's $t$-test). I Quantitative analysis of calcium mineralization in MSCs ( $n=3$, by two-tailed Student's $t$-test). $\mathbf{m}$ Quantitative RT-PCR results of mRNA expression of Runx2, Sp7, Colla1, Alp, and Bglap in MSCs treated with vehicle/ $\alpha K G$ ( $n=3$, by two-tailed Student's $t$-test). Data are expressed as mean \pm SEM.

envelope architecture (Fig. $5 c, d$ ), as confirmed by the immunofluorescence of progerin ${ }^{28}$ (aberrant prelamin A isoform). Phosphorylation of Ser-139 of histone H2A.X molecules ( $\gamma$-H2A.X), a common indicator of DNA damage ${ }^{29}$, was also reduced by aKG treatment (Fig. 5e, f). In addition, as revealed by senescenceassociated $\beta$-galactosidase (SA- $\beta$-gal) staining, the percentage of positive cells was decreased in the $\alpha \mathrm{KG}$ group (Fig. $5 \mathrm{~g}, \mathrm{~h}$ ).

Next, we examined the ROS levels in MSCs 3 days after aKG treatment. The results showed a remarkable reduction in ROS levels in aKG-treated MSCs compared to the controls (Fig. 5i). We then applied $\mathrm{H}_{2} \mathrm{O}_{2}$ to exacerbate cell senescence and confirmed that $\alpha \mathrm{KG}$ was capable of alleviating MSCs senescence induced by ROS (Fig. 5j, k).

aKG downregulates the global H3K9me3 and H3K27me3 levels. Given the role of histone modifications in the aging process ${ }^{30-32}$, we sought to investigate whether aKG exerted its function by modifying histone marks. Western blot analysis showed that $\alpha \mathrm{KG}$ supplementation significantly reduced $\mathrm{H} 3 \mathrm{~K} 9 \mathrm{me} 3$ and $\mathrm{H} 3 \mathrm{~K} 27 \mathrm{me} 3$ levels in the aged MSCs isolated from 18-month-old mice, while levels of $\mathrm{H} 3 \mathrm{~K} 4 \mathrm{me} 3, \mathrm{H} 3 \mathrm{~K} 9 \mathrm{ac}$, and $\mathrm{H} 3 \mathrm{~K} 27 \mathrm{ac}$ remained unchanged (Fig. 6a). The downregulation of $\mathrm{H} 3 \mathrm{~K} 9 \mathrm{me} 3$ and $\mathrm{H} 3 \mathrm{~K} 27 \mathrm{me} 3$ was confirmed by immunofluorescence staining (Fig. 6b-e).

Next, we performed immunofluorescence staining of LepR, a bone marrow MSC marker ${ }^{33,34}$, on vertebral sections from aged mice. aKG administration increased $\mathrm{LepR}^{+}$cell pools in vivo, indicating a relatively enlarged MSCs population in the bone marrow (Fig. 6f, g). Notably, the percentage of MSCs which were double labeled with LepR and $\mathrm{H} 3 \mathrm{~K} 9 \mathrm{me} 3$ was significantly lower compared to the controls (Fig. 6h, i). The percentage of H3K27me3-stained $\mathrm{LepR}^{+}$MSCs was also reduced by aKG administration (Fig. 6j, k). 

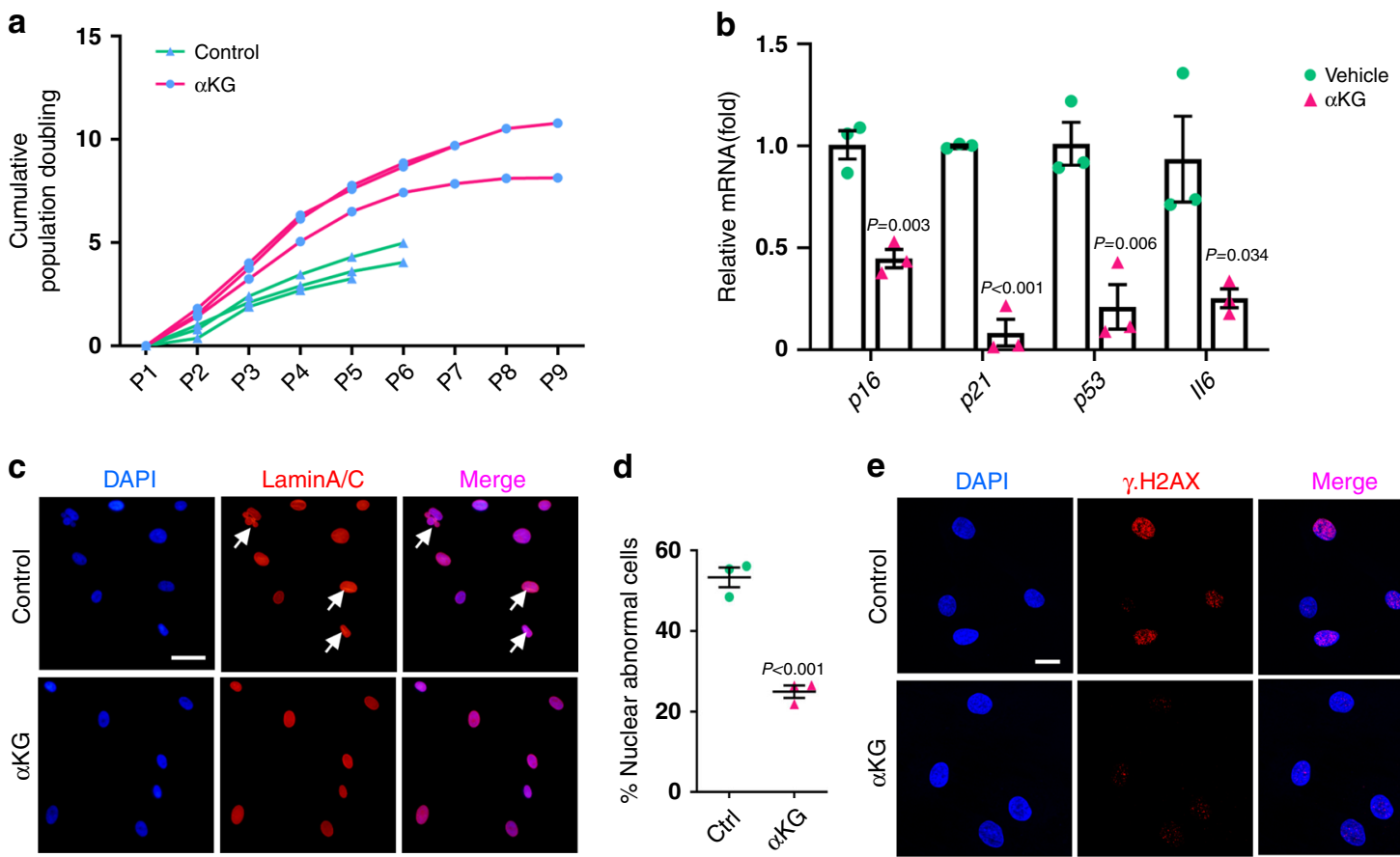

d

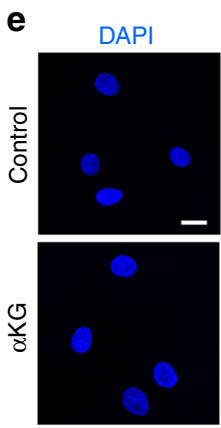

$\gamma \cdot \mathrm{H} 2 \mathrm{AX}$
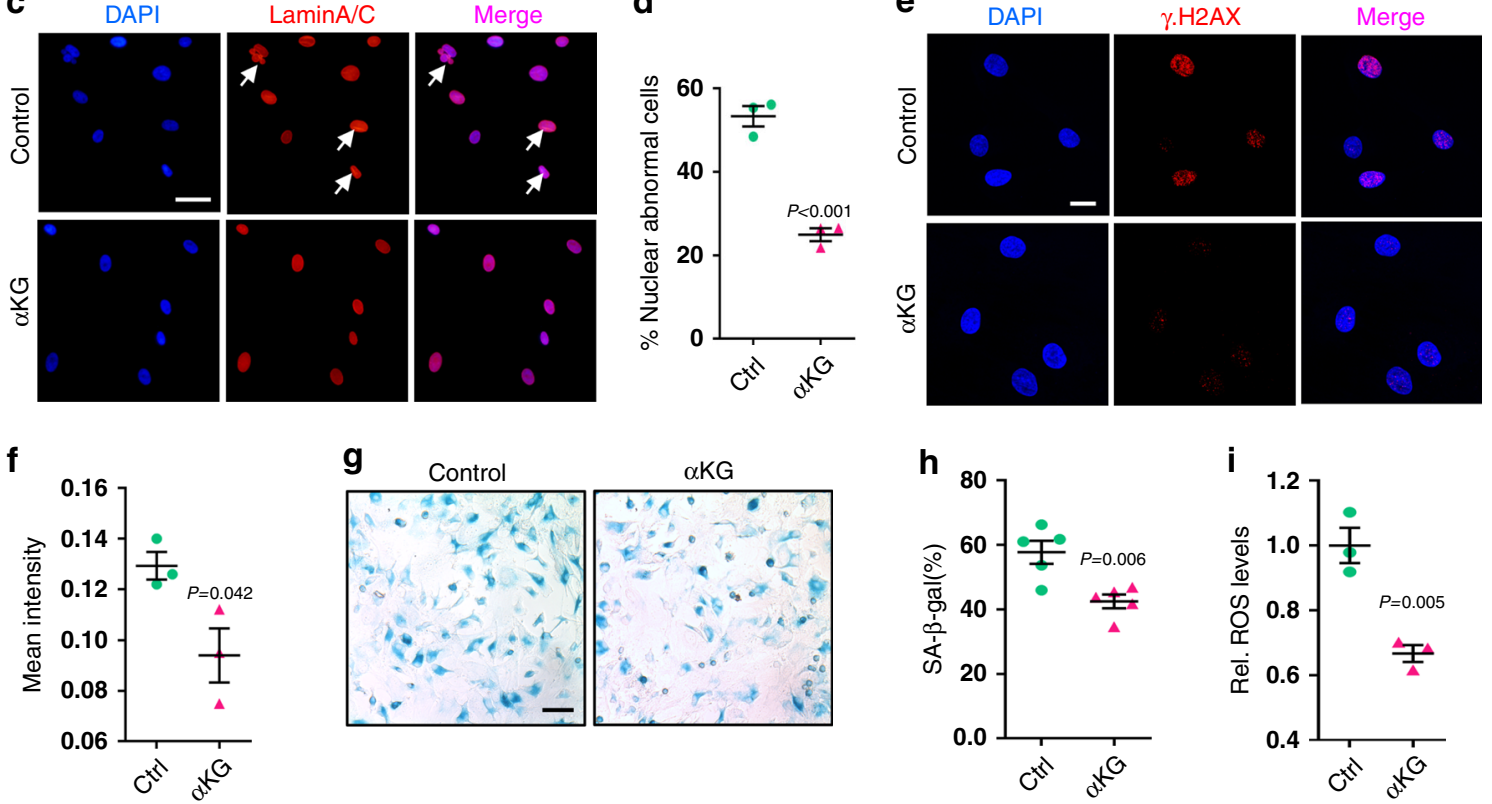

h
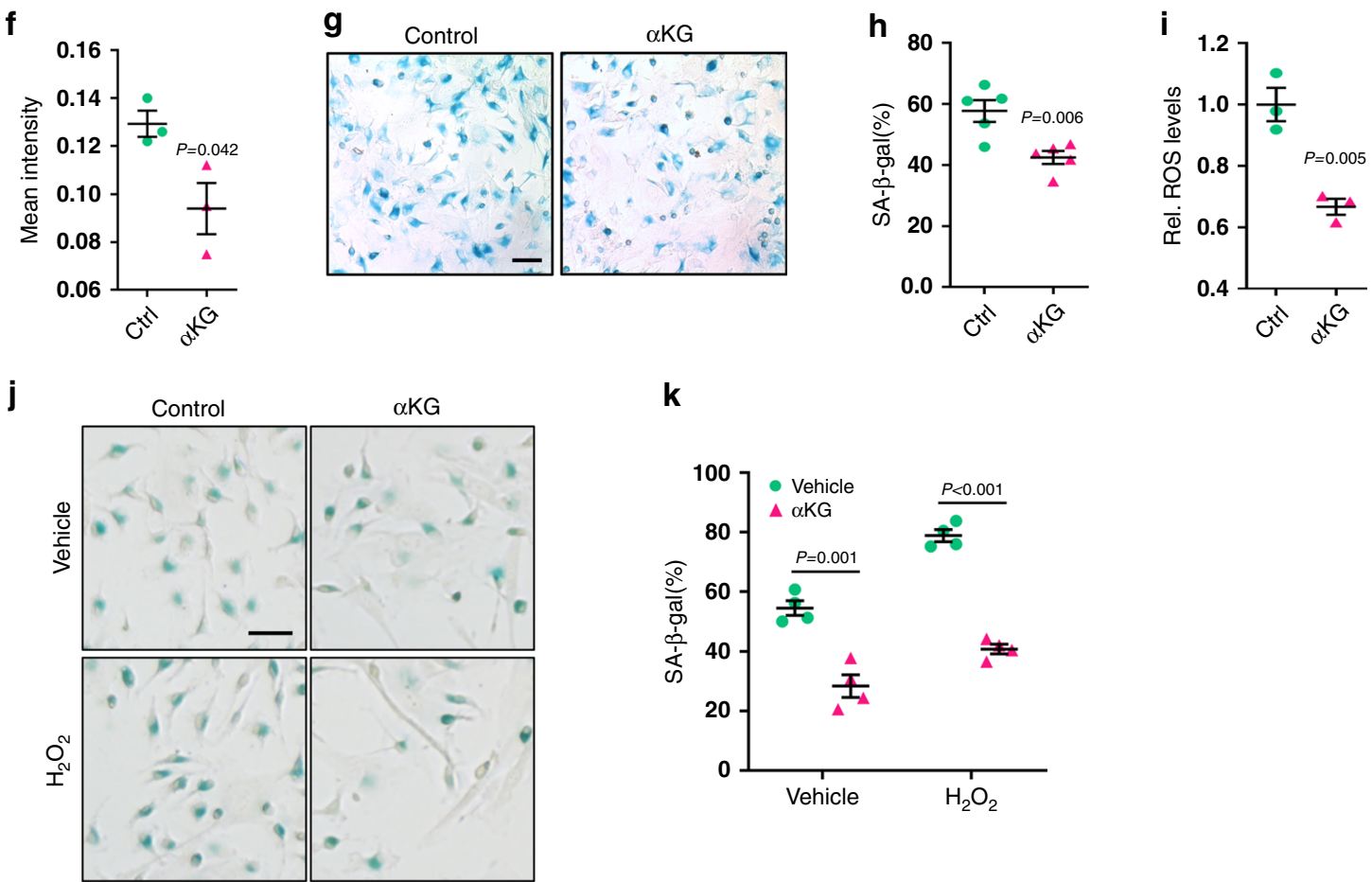

k

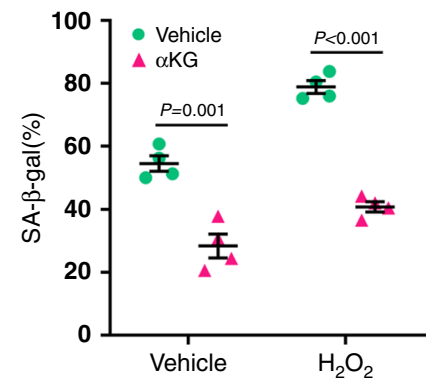

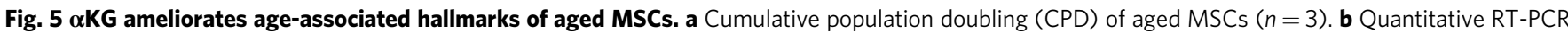
results of senescence-associated genes $(p 16, p 21, p 53,116)$ in aged MSCs $(n=3)$. Cells were treated with 2 mM $\alpha$ KG for 3 days. c, $\mathbf{d}$ Immunofluorescence of Lamin A/C and quantification of nuclear abnormality in MSCs $(n=3)$. White arrows indicate blebbing in the nuclear envelope. Scale bar, $50 \mu \mathrm{m}$.

e, $\mathbf{f}$ Immunofluorescence of $\gamma \mathrm{H} 2 \mathrm{~A}$.X and quantification of mean density in MSCs $(n=3)$. Scale bar, $20 \mu \mathrm{m}$. $\mathbf{g}$, $\mathbf{h}$ Representative images of senescenceassociated $\beta$-galactosidase (SA- $\beta$-gal) staining of aged MSCs and quantification of positive cells $(n=5)$. Scale bar, $50 \mu \mathrm{m}$. i ROS levels in aged MSCs ( $n=$ 3) were decreased after $\alpha$ KG supplement for 3 days. $\mathbf{j}$ SA- $\beta$-gal staining of aged MSCs stimulated with $\mathrm{H}_{2} \mathrm{O}_{2}$. Scale bar, $50 \mu \mathrm{m}$. $\mathbf{k}$ Quantification of SA- $\beta$-gal positive MSCs $(n=4)$. Data are expressed as mean \pm SEM. The $P$ values were calculated by two-tailed Student's $t$-test, except $\mathbf{k}$, which was analyzed by two-way ANOVA with Sidak's multiple comparisons test.

aKG regulates BMP pathway through histone methylations. Next, we performed RNA sequencing (RNA-seq) to examine transcriptome profiles in aged MSCs treated with or without aKG. Gene set enrichment analysis (GSEA) validated the upregulated expression of osteogenic marker genes (Fig. 7a). Of note, we find a significantly elevated expression of BMP-regulated genes, including Bmp2 and Bmp4 (Fig. 7b, c). We also found that the expression of Nanog, a master regulator of stem cell potency ${ }^{35}$, was increased (Fig. 7c).

Next, we examined the effect of aKG on accumulations of $\mathrm{H} 3 \mathrm{~K} 9 \mathrm{me} 3$ and $\mathrm{H} 3 \mathrm{~K} 27 \mathrm{me} 3$ at promoter regions of Bmp2, Bmp4, 
a

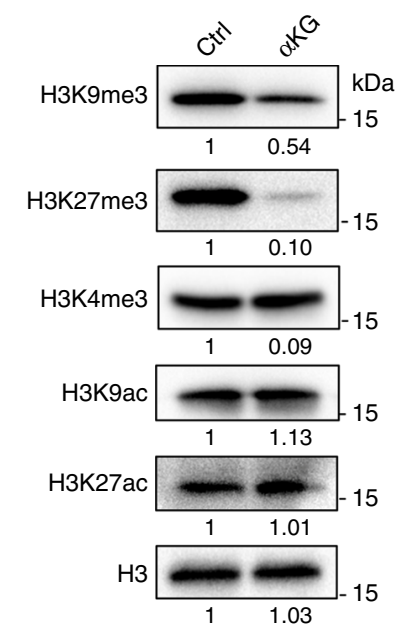

f

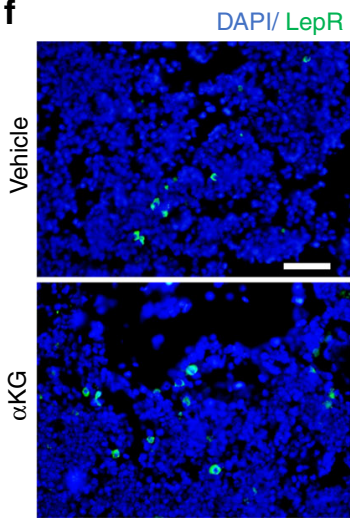

i

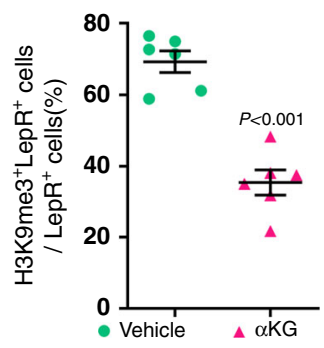

b
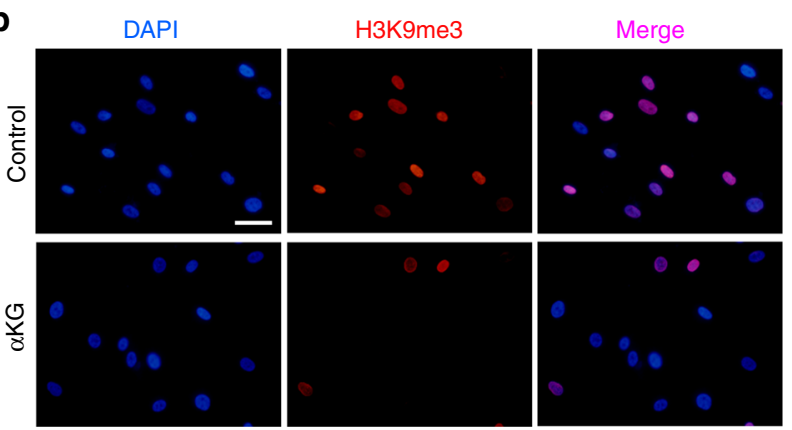

C
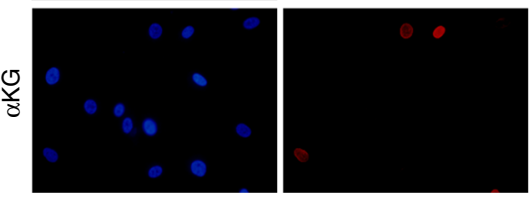

d
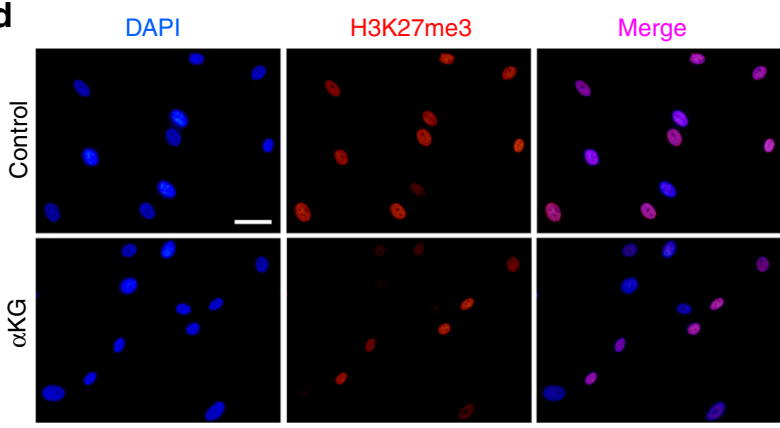

h
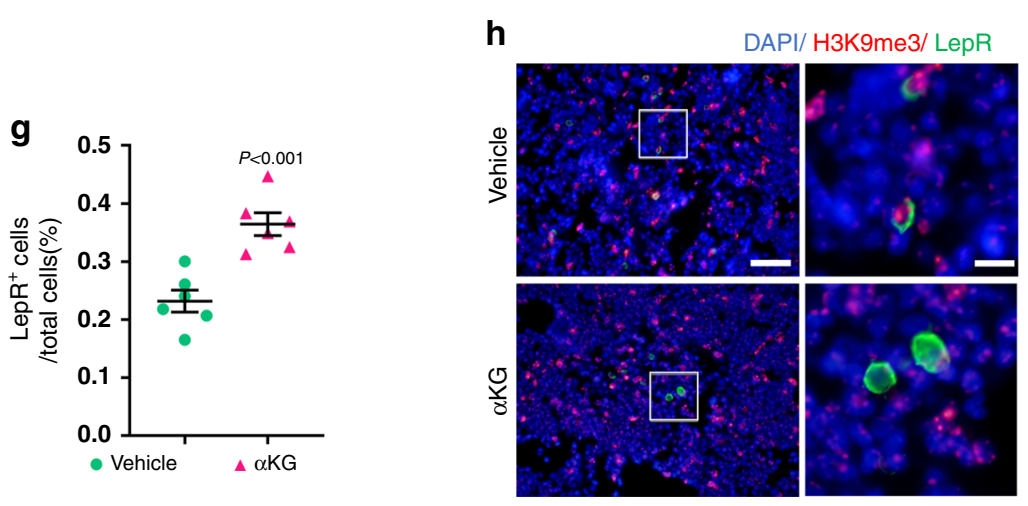

DAPI/ H3K27me3/ LepR

j

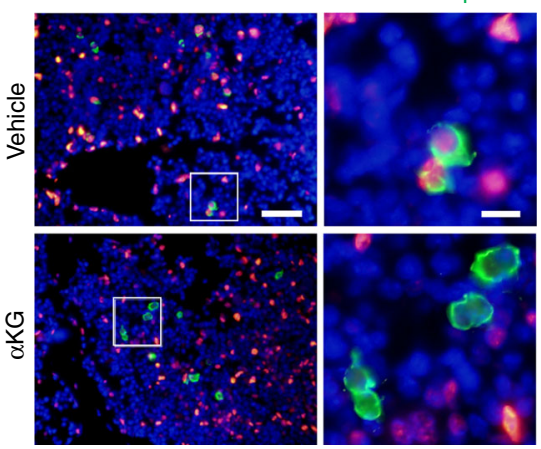

e
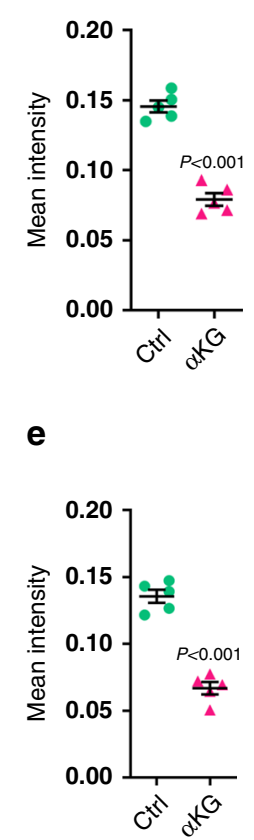

e

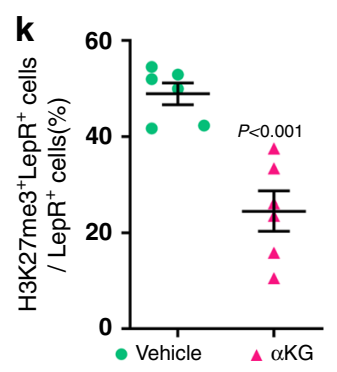

Fig. 6 жKG decreases H3K9me3 and H3K27me3 abundance in aged MSCs. a Western blot analyses of H3K9me3, H3K27me3, H3K4me3, H3K9ac, and H3K27ac in aged MSCs treated with $2 \mathrm{mM} \alpha \mathrm{KG}$ for 3 days. b, c Immunofluorescence staining of H3K9me3 and quantification ( $n=5$ ). Aged cells were treated with $2 \mathrm{mM} \alpha \mathrm{KG}$ for 3 days. Scale bar, $50 \mu \mathrm{m}$. d, e Immunofluorescence staining of H3K27me3 and quantification $(n=5)$. Scale bar, $50 \mu \mathrm{m}$. f, $\mathbf{g}$ Immunofluorescence staining and quantification of LepR ${ }^{+}$cells in vertebrae from aged mice fed with $\alpha K G$ for a month $(n=6)$. Scale bar, $50 \mu \mathrm{m}$. $\mathbf{h}$, i Double staining and quantification of LepR-positive and H3K9me3-positive MSCs in vertebrae from aged mice fed with $\alpha$ KG for a month ( $n=6)$. Scale bar, $50 \mu \mathrm{m}$ (left) or $10 \mu \mathrm{m}$ (right). j, $\mathbf{k}$ Double staining and quantification of LepR-positive and H3K27me3-positive MSCs in vertebrae from aged mice fed with $\alpha K G$ for a month $(n=6)$. Scale bar, $50 \mu \mathrm{m}$ (left) or $10 \mu \mathrm{m}$ (right). Data are presented as mean \pm SEM. The $P$ values were calculated by two-tailed Student's t-test. 
a

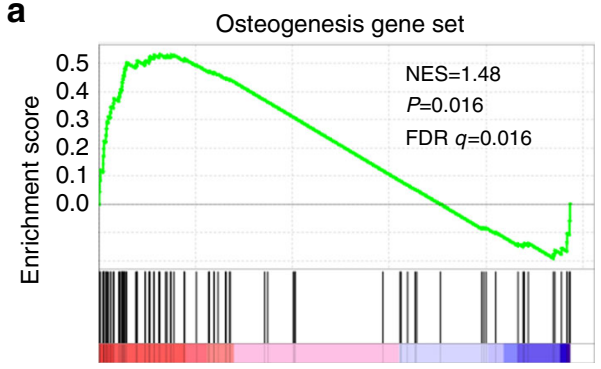

b

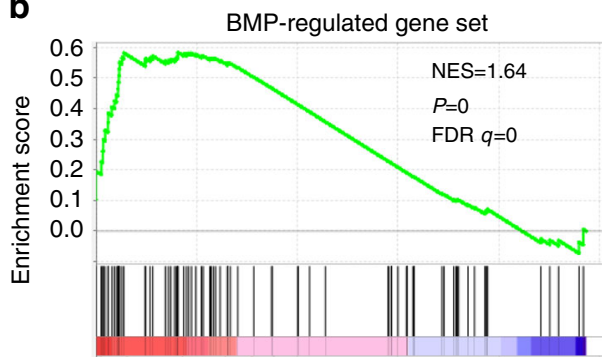

d

Bmp2

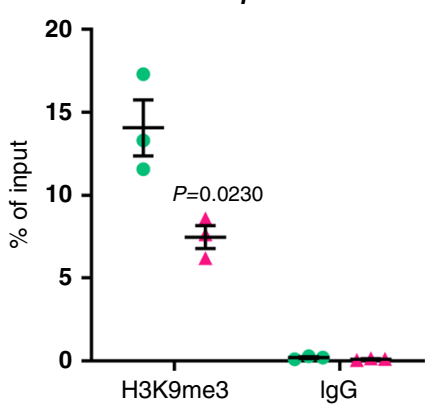

e

Bmp2
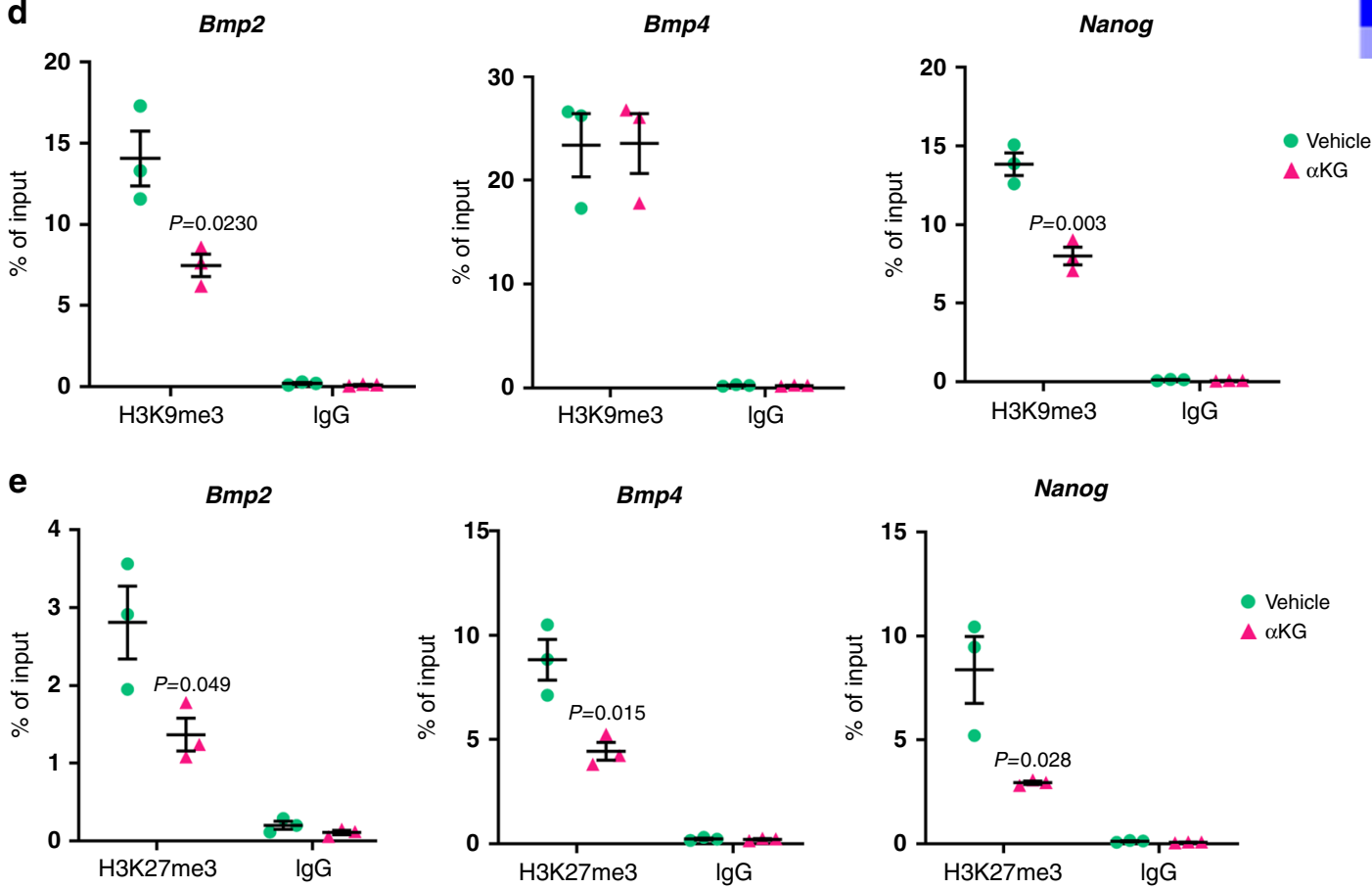

$\mathbf{f}$

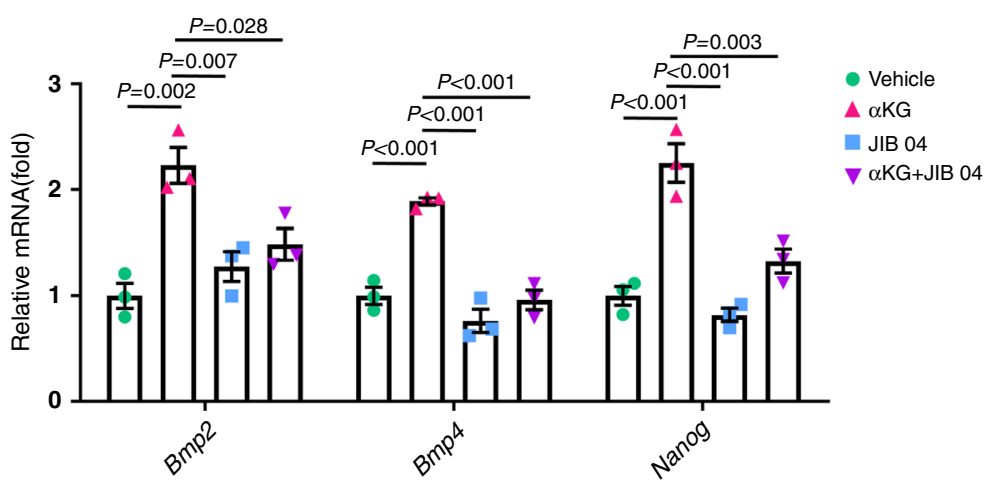

C Ctrl $\alpha K G$

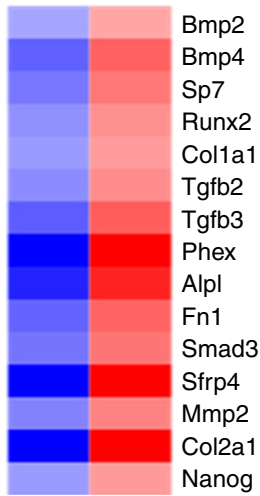

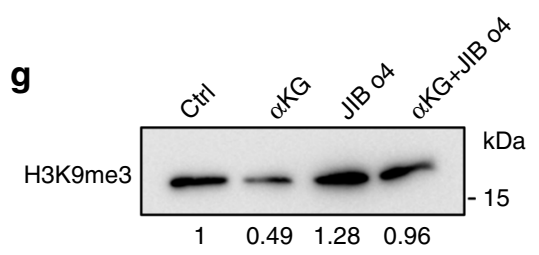
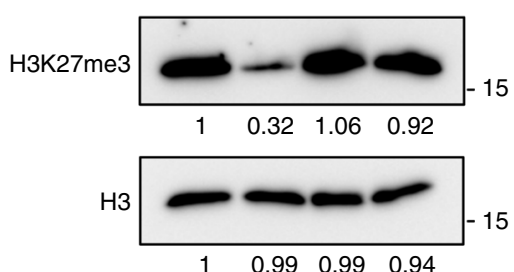

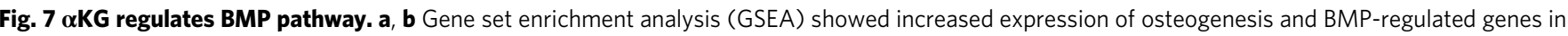
$\alpha K G$-treated MSCs. Cells isolated from aged mice were treated with $2 \mathrm{mM} \alpha \mathrm{KG}$ for $48 \mathrm{~h}$. $\mathbf{c}$ Heatmap of representative genes. $\mathbf{d}$ ChIP-qPCR assay revealed that $\alpha K G$ decreased the occupancy of H3K9me3 in the promoter regions of Bmp2 and Nanog ( $n=3$, by two-tailed Student's $t$-test). e ChIP-qPCR showed that $\alpha K G$ decreased the occupancy of H3K27me3 in the promoter regions of Bmp2, Bmp4, and Nanog ( $n=3$, by two-tailed Student's $t$-test). $\mathbf{f}$ Quantitative RT-PCR ( $n=3$, by one-way ANOVA with Tukey's post hoc test). Histone demethylases inhibitor JIB 04 prohibited the effect of $\alpha$ KG on promoting Bmp2, Bmp4, and Nanog expression. $\mathbf{g}$ Western blot analyses of H3K9me3 and H3K27me3 in aged MSCs after supplementation of $\alpha$ KG and JIB 04. Data are shown as mean \pm SEM. 
and Nanog by chromatin immunoprecipitation (ChIP) assay. The enrichment of $\mathrm{H} 3 \mathrm{~K} 9 \mathrm{me} 3$ at the promoter regions of $\mathrm{Bmp} 2$ and Nanog were reduced in the aged MSCs isolated from 18-monthold female mice when treated with aKG (Fig. 7d). Consistently, aKG treatment reduced H3K27me3 occupancy at the promoters of Bmp2 and Bmp4, as well as in the promoter region of Nanog (Fig. 7e).

Notably, the mRNA levels of Bmp2, Bmp4, Nanog in aged MSCs were upregulated after $\alpha \mathrm{KG}$ treatment, while this effect was largely abolished after the supplement of JIB 04 (Fig. 7f), a selective inhibitor of Jumonji histone demethylases ${ }^{36}$. Meanwhile, the mRNA expression levels of $\mathrm{H} 3 \mathrm{~K} 9$ me3-modifying and H3K27me3-modifying enzymes remained unchanged after aKG treatment (Supplementary Fig. 7a, b). Besides, the application of $1 \mu \mathrm{M} \mathrm{JIB} 04$ for 3 days counteracted the downregulation effects of aKG on $\mathrm{H} 3 \mathrm{~K} 9 \mathrm{me} 3$ and $\mathrm{H} 3 \mathrm{~K} 27 \mathrm{me} 3$ at protein level via western blot analyses (Fig. 7g). Those findings support that the effect of aKG on MSCs aging relies, at least partially, on the regulation of histone modifications.

\section{Discussion}

As the aging population growing worldwide, discovering therapies to rejuvenate the deteriorated physiological functions and ameliorate the age-associated symptoms has become an intriguing issue in scientific fields $s^{4,37}$. $\alpha \mathrm{KG}$ is a crucial intermediate of the Kreb Cycle that is recently reported to have potential antiaging effects. The data from in vitro studies support that $\alpha \mathrm{KG}$ plays roles in maintaining the pluripotency of $\mathrm{ESCs}^{21}$, and accelerating the primordial differentiation of primed human pluripotent stem cells and germ cells ${ }^{38,39}$. Furthermore, Huang et al. revealed that $a K G$ treatment successfully extended the lifespan of elegans ${ }^{22}$. Specifically, Lin et al. reported that maintenance of aKG levels via caloric restriction played a crucial role in preserving brain physiology in aged rats ${ }^{40}$. Another in vivo study showed that dietary supplementation of aKG stabilized redox state and improved blood vessel elasticity in aged mice ${ }^{41}$. Moreover, Cynober et al. noted that, ornithine-ketoglutarate improved appetite, increased body weight gain, and accelerated wound healing, thus improving the clinical outcome of chronic malnutrition in elderly patients ${ }^{42,43}$.

To our knowledge, this is the first study that investigates the effect of aKG on skeleton aging using a model of age-related osteoporosis. A previous study reported that, aKG level in human serum experienced a notable decline upon aging ${ }^{44}$. Likewise, circulatory aKG concentration was found to be reduced in middle-aged mice (10-month-old) compared with young mice (2month-old $)^{45}$. Consistently, we observed such reduction in aged rodents compared to younger organisms. aKG supplementation successfully compensates for this decline, in which case provides the rationality for us to explore the potential therapy of skeleton aging through $\alpha \mathrm{KG}$ administration.

In this study, we illustrated that supplementation of $\alpha \mathrm{KG}$ in drinking water not only increased the bone mass of physiologically aged mice, but also attenuated the rapid bone loss in adult mice. We showed that the female C57BL/6J mice exhibited a 77\% reduction of femoral trabecular bone volume from 2-month old to 6-month old, which is close to the data of Glatt et al. ${ }^{23}$, but higher than some other reports. Previous studies show cancellous bone loss begins in early adulthood in C57BL/6 mice (2-3month-old), earlier than that of BMD $23,24,46$. Notably, different mouse strains exhibit different patterns in bone $\operatorname{loss}^{47-49}$. C57BL/ $6 \mathrm{~J}$ mice experience bone loss as early as 2 months of age, while $\mathrm{BALB} / \mathrm{c}$ mice wouldn't show significant reduction until 7 months ${ }^{50}$. Female mice also undergo a sharper decline in BV/ TV. C57BL/6J female mice may experience a reduction of about
$70 \%$ femoral trabecular bone from 2-month old to 6-month old 23 , while male mice lose $25-35 \%$ of the value ${ }^{23,24}$. In addition, the methods of Micro CT scanning and analysis may affect the data. In this study, we used a Scanco $\mu$ CT50 scanners. For long bone, trabecular bone was analyzed starting from $160 \mu \mathrm{m}$ away from the growth plate and extending $1200 \mu \mathrm{m}$ proximally. As for vertebra, the region $160 \mu \mathrm{m}$ below the cranial and above the caudal growth plate in the vertebral body was selected for analysis.

In the past few decades, scientific perspectives on the pathogenesis of osteoporosis have undergone a transformation from estrogen-centric to aging-centric ${ }^{51}$. The intrinsic cause of osteoporosis is considered to be the age-related changes in multiple organs and tissues (immune system, skeleton system, and endocrine system, etc.), particularly the dysregulation of MSC lineage commitment in the aged bone environment ${ }^{52}$. In this study, we proved the protective effects of aKG in MSCs aging. Of note, deteriorated capacity of proliferation, migration, and osteogenesis of MSCs towards aging is well-characterized by previous studies $^{26,27}$. Here we found that, aKG could largely maintain the proliferation vitality, enhanced the migration of MSCs in vitro, and promoted osteogenic differentiation of aged MSCs. Moreover, with the treatment of $\alpha K G$ in vitro, the lifespan of aged MSCs was extended and the senescence-associated hallmarks of MSCs were significantly downregulated, including the mRNA level of SA genes ( $p 16, p 21, p 53$, and Il6), markers of nuclear architecture (Lamin A/C) and DNA damage marker $\gamma$-H2A.X, and SA- $\beta$-gal activities. As aging increases the susceptibility of MSCs towards $\mathrm{ROS}^{53}$, our data reveal a restrained ROS level and cell senescence upon $\alpha \mathrm{KG}$ treatment, either in vehicle condition or under the exacerbated ROS stress induced by $\mathrm{H}_{2} \mathrm{O}_{2}$. These results are consistent with previous studies in different cell types and organisms ${ }^{54}$.

aKG has been reported to bind and block the mitochondrial ATP synthase, leading to the inhibition of mechanistic target of rapamycin (mTOR) signaling, through which aKG extended worm lifespan ${ }^{22,55}$. It is believed that, whether aKG favors ESCs and pluripotent stem cells (PSCs) towards self-renewal or differentiation largely relies on the pluripotent stage, which is affected by oxidative phosphorylation (OXPHOS) level, induction circumstance, etc ${ }^{38,56,57}$. However, TeSlaa et al. showed that aKG did not promote differentiation of primed PSCs via inhibiting ATP synthase. Instead, the role of aKG in epigenetic regulation was suggested to be responsible for favoring the naive state of PSCs or ESCs, or accelerating induced primed PSC differentiation ${ }^{21,38}$.

Epigenetic alterations are regarded as hallmarks of cellular senescence or organismal aging ${ }^{58}$. And histone modifications are documented as an essential component ${ }^{59,60}$. Our data show aKG relieves the overall burden of $\mathrm{H} 3 \mathrm{~K} 9 \mathrm{me} 3$ and $\mathrm{H} 3 \mathrm{~K} 27 \mathrm{me} 3$, with little effects on levels of $\mathrm{H} 3 \mathrm{~K} 4 \mathrm{me} 3, \mathrm{H} 3 \mathrm{~K} 9 \mathrm{ac}$, and $\mathrm{H} 3 \mathrm{~K} 27 \mathrm{ac}$. H3K9me3 and H3K27me3 are two critical histone modifications that are closely associated with cell senescence and organismal aging, and age-associated osteoporosis as well ${ }^{61-63}$. Previous in vitro studies showed that a relatively low methylation level of $\mathrm{H} 3 \mathrm{~K} 9 \mathrm{me} 3$ and $\mathrm{H} 3 \mathrm{~K} 27 \mathrm{me} 3$ is a linchpin to maintain the pluripotency of $\mathrm{ESCs}^{21}$. Ye et al. have reported that in the bone marrow of aged mice, the overall accumulations of $\mathrm{H} 3 \mathrm{~K} 9 \mathrm{me} 3^{+}$ and $\mathrm{H} 3 \mathrm{~K} 27 \mathrm{me} 3^{+}$cells are significantly elevated compared with the young controls in vivo ${ }^{32}$. Here, both our in vitro and in vivo data show that the number of $\mathrm{H} 3 \mathrm{~K}_{9} \mathrm{me}^{+}$and $\mathrm{H} 3 \mathrm{~K} 27 \mathrm{me}^{+}$ MSCs were reduced after aKG administration.

aKG is believed to serve as a co-substrate for $\mathrm{Fe}(\mathrm{II}) / \mathrm{aKG}$ dependent dioxygenases, which are widely present in all living organisms $s^{64,65}$. These enzymes play important roles in various biological processes including the post-translational modification of collagen, fatty acid metabolism, oxygen sensing, DNA and 
RNA repair, demethylations related to epigenetic regulation ${ }^{66}$. Of note, the JMJC family of histone demethylases can be classified into aKG-dependent dioxygenases, using aKG as an indispensable cofactor ${ }^{67,68}$. As histone methylation status is manipulated by the counteraction of histone methyltransferases and demethylases, the JMJC family of histone demethylases has been reported as the crucial regulator of MSC differentiation ${ }^{69}$. Here, we demonstrate downregulation of $\mathrm{H} 3 \mathrm{~K} 9 \mathrm{me} 3$ and $\mathrm{H} 3 \mathrm{~K} 27 \mathrm{me} 3$, without affecting the expression of histone-modifying enzymes, which implies a more active status of histone demethylases upon aKG administration.

In summary, we demonstrate that aKG protects age-related osteoporosis and rejuvenates aged MSCs. It reduces overall H3K9me3 and H3K27me3 levels, and their occupancy at promoters of Bmp2, Bmp4, and Nanog, highlighting the therapeutic potential of $a \mathrm{KG}$ in age-related osteoporosis.

\section{Methods}

Animals. C57BL/6J mice and Sprague Dawley rats were purchased from the Chengdu Dossy Experimental Animals CO. LTD. Animals were randomly allocated into either control or aKG group and housed in specific pathogen-free facilities under a 12 -h light and 12 -h dark cycle. Temperature $\left(23 \pm 2{ }^{\circ} \mathrm{C}\right)$ and humidity $(55 \%)$ were held constant in animal housing. All animals were allowed free access to food and clean water in the absence or presence of $\alpha \mathrm{KG}$ (K1128, Sigma). Both the vehicle water and aKG solution were adjusted to 7.3 by the addition of $\mathrm{NaOH}^{16,17}$. Approvals for all the protocols were obtained from the Subcommittee on Research and Animal Care (SRAC) of Sichuan University.

$\boldsymbol{\mu C T}$ analysis. Bone tissues (femur, tibia, and vertebra) were dissected and fixed in $10 \%$ paraformaldehyde for $48 \mathrm{~h}$ and stored in $70 \%$ ethanol at $4{ }^{\circ} \mathrm{C}$ before being processed. The microCT imaging system ( $\mu$ CT50, SCANCO Medical) was applied to evaluate trabecular bone and bone regeneration. The specimens were well placed in a cylindrical holder ( $14 \mathrm{~mm}$ in diameter) with the long axis of the bone tissues perpendicular to the X-ray beam and a spatial resolution of $8 \mu \mathrm{m}(55 \mathrm{kV}, 114 \mathrm{~mA}$, $500 \mathrm{~ms}$ integration time). Thereafter, volumetric reconstructions and analyses were performed by built-in software. For trabecular bone analysis, the region of interest (ROI) was selected according to the anatomical structure of those bone tissues. One hundred and fifty slices were chosen as ROIs for long bone (distal femur and tibia), starting from 20 slices beneath the growth plates. As for vertebrae (L4), around $300-400$ slices of the vertebral body were selected as the ROIs for analysis $(160 \mu \mathrm{m}$ below the cranial and above the caudal growth plate regions). Bone volume fraction (BV/TV), trabecular number (Tb.N), trabecular separation (Tb.Sp), and trabecular thickness (Tb.Th) were calculated within the ROIs. For bone regeneration analysis, ROI was defined as a cylindrical area situating at the initial bone defect. Bone volume fraction (BV/TV) and the bone mineral density (BMD) within the delimited ROIs were evaluated.

Histomorphometric analyses. Processing of undecalcified bone specimens and cancellous bone histomorphometry were performed as described ${ }^{25,70}$. Bone specimens (femur, tibia, and vertebra) were dehydrated and embedded in methylmethacrylate. Sections ( $5 \mu \mathrm{m}$ in thickness) were prepared using a microtome (\#RM2235, Leica) microtome and were stained by the von Kossa/nuclear fast red method. For hematoxylin and eosin (HE) and tartrate-resistant acid phosphatase (TRAP) staining, bone specimens were decalcified in 10\% EDTA for 2 weeks at room temperature. After serial dehydration in a series of ethanol (70-100\%), samples were embedded in paraffin. Sections ( $5 \mu \mathrm{m}$ in thickness) were processed by a microtome (\#RM2235, Leica) ready for staining. Histomorphometric measurements were performed using OsteoMeasure software (OsteoMetrics, Decatur, GA ${ }^{71}$.

Cell culture. Primary MSCs were isolated from aged C57BL/6J mice at 18 months old by flushing the bone marrow of tibiae and femurs according to the published protocol ${ }^{72}$. Cells were cultured in minimum essential medium $\alpha$ ( $\alpha$-MEM) supplemented with $10 \%$ heat-inactivated fetal bovine serum (Gibco) and $1 \%$ penicillin/ streptomycin (HyClone), with or without supplementation of cell-permeable $\alpha \mathrm{KG}$ (K1128, Sigma). MSCs were grown on $100 \mathrm{~cm}^{2}$ dish (Corning) at $37^{\circ} \mathrm{C}$ in an atmosphere of $5 \% \mathrm{CO}_{2}$. MSCs were passaged at $90 \%$ confluence ${ }^{73}$.

Osteogenic induction and alkaline phosphatase activity assay. In vitro osteogenesis of MSCs was induced using the osteogenic differentiation medium, containing $50 \mu \mathrm{g} / \mathrm{ml}$ ascorbic acid, $5 \mathrm{mM} \beta$-glycerophosphate, $100 \mathrm{nM}$ dexamethasone (all from Sigma) ${ }^{25}$, supplemented with or without $2 \mathrm{mM}$ aKG.

On day 10 , cells were fixed with $4 \%$ polyoxymethylene for $30 \mathrm{~min}$. After washing cells with PBS for three times, cells were incubated at $37^{\circ} \mathrm{C}$ with $0.1 \mathrm{M}$ Tris buffer ( $\mathrm{pH}$ 9.3) consisting 0.25\% naphthol AS-BI phosphate (\#N2125, Sigma) and $0.75 \%$ Fast Blue BB (D9805, Sigma). Images of stained plates were obtained using a scanner (\#V330, EPSON). Quantification of alkaline phosphatase (ALP) activity was performed using a commercial kit, according to the instructions (\#P0321, Beyotime). And the optical density was examined via spectrophotometer (Thermo Fisher Scientific) at $405 \mathrm{~nm}$.

Mineralization assay and Van Gieson staining. Alizarin Red S (ARS) staining was performed as described ${ }^{71}$. Briefly, cells were fixed after 21-day induction of osteogenic differentiation with $4 \%$ polyoxymethylene for $30 \mathrm{~min}$. After washing cells with PBS for three times, cells were stained with $1 \%$ Alizarin red S ( $\mathrm{pH} 4.2$, Sigma-Aldrich) for $20 \mathrm{~min}$. Images of stained plates were obtained using a scanner (V330, EPSON). For mineralization quantification, mineralized matrix stained with alizarin red were destained with $10 \%$ cetylpyridinium chloride for 30 min continuous gentle shaking. The calcium concentration was evaluated by detecting the optical density at $562 \mathrm{~nm}$ (Thermo Fisher Scientific), and converting those data using the standard calcium curve in the same solution.

Van Gieson Staining was performed using a commercial kit (G1338, Solarbio), according to manufacturer's instruction.

Bone defect surgery. The rodent bone defect model was established through the surgery on the long bone of aged rats. In brief, aged female rats at 24-month-old were anesthetized through intraperitoneal injection of a mixture of ketamine $\left(100 \mathrm{mg} \mathrm{kg}^{-1}\right)$ and xylazine $\left(10 \mathrm{mg} \mathrm{kg}^{-1}\right)$. Besides, buprenorphine $\left(0.05 \mathrm{mg} \mathrm{kg}^{-1}\right)$ was administrated before operation to minimize suffering and pain. The anteriordistal surfaces of the femurs were exposed by blunt dissection of the quadriceps after skin incision. A $1.4 \mathrm{~mm}$ defect was created with a round bur (Komet, Germany) operating at 10,000 r.p.m. under irrigation of saline, and then ample saline was used to rinse off bone fragments. Muscles were stitched using interrupted 5-0 Monocryl sutures and skin closures were completed subsequently with 3-0 sutures. Bone tissues were collected 2 or 4 weeks after surgery. Upon specimen harvest, femoral samples were fixed in $4 \%$ paraformaldehyde for $48 \mathrm{~h}$ at $4{ }^{\circ} \mathrm{C}$ and were transferred into $70 \%$ ethanol and stored at $4{ }^{\circ} \mathrm{C}$ for further experiments.

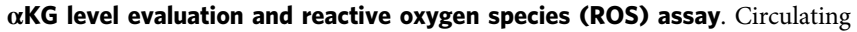
$\alpha K G$ levels in serum and intracellular $\alpha K G$ levels were measured by using an $\alpha K G$ assay kit (ab83431, Abcam) according to the manufacturer's instructions. After 3 days of treatment with $\alpha \mathrm{KG}$, ROS levels in cells were measured using cellular ROS detection kit (ab186027, Abcam) following the manufacturer's instructions.

Proliferation assay and apoptosis assay. MSCs were treated with different concentrations of $\alpha \mathrm{KG}(0 / 2 / 4 / 8 \mathrm{mM})$ for 3 days, and then were plated on 8 -well culture slides overnight to allow for attachment. For 5-ethynyl-2 $2^{\prime}$-deoxyuridine (EdU) incorporation assay, cells were cultured in the presence of $10 \mu \mathrm{M}$ EdU and different concentrations of $a \mathrm{KG}(0 / 2 / 4 / 8 \mathrm{mM})$ for $24 \mathrm{~h}$, and then fixed and permeabilized for imaging according to the manufacturer's instructions (C10337, Invitrogen).

Apoptotic cells were detected using FITC Annexin V Apoptosis Detection Kit I according to the manufacturer's instructions (\#556547, BD Biosciences).

Colony formation assay. For colony formation assay, MSCs were seeded at 200 cells per well in 6-well plates with or without $2 \mathrm{mM} \alpha \mathrm{KG}$. Medium was changed every 3 days, and cells were fixed and stained with $0.5 \%$ crystal violet in $98 \%$ ethanol on day 10. Colonies containing more than 50 cells were counted in each well.

Migration assay. In vitro migration activity of MSCs was evaluated via scratch assay and transwell assay. For the scratch assay, MSCs were cultured in 6-well plates until reaching confluence and began to be starved $24 \mathrm{~h}$ before scratching. Cells were scraped rapidly across the center of the plates via a pipette tip $(20-200 \mu \mathrm{l})$. Photographs were taken under an inverse phase-contrast microscope at $0 / 9 / 18 \mathrm{~h}$ after cell scratch, and the average width of the scratched gap was measured in triplicate wells by the ImageJ software (v.1.6.0). For transwell assay, the transwell inserts with $8 \mu \mathrm{m}$ membrane (\#3422, Corning) were plated in the wells of 24 -well plates. Six hundred microliter of $10 \%$ FBS medium with or without aKG was added to the lower chamber, and MSCs $\left(5 \times 10^{4}\right.$ cells/well $)$ were seeded into the upper chamber in a serum-free medium. After $12 \mathrm{~h}$ incubation at $37^{\circ} \mathrm{C}$, medium in the lower and upper chamber was discarded, and the cells lining in the upper side of the membrane were removed by cotton swabs. MSCs migrated to the lower surface of the membrane were fixed with $4 \%$ paraformaldehyde for $30 \mathrm{~min}$ and stained with $0.05 \%$ crystal violet for $1 \mathrm{~h}$. Randomly selected and photographed five fields for each sample at $\times 200$ magnification under a microscope. The number of stained cells per field was counted and calculated.

Lifespan analysis. MSCs obtained from aged mice (18-20 months old) were seeded $\left(1 \times 10^{4}\right.$ cells $\left./ \mathrm{cm}^{2}\right)$ in $100 \mathrm{~mm}$ dishes. The adherent cells were counted after 3-4 days to quantify the initial number. At $90 \%$ confluence, the cells were digested in trypsin (HyClone, GE), counted and reseeded at $5 \times 10^{3}$ cells $/ \mathrm{cm}^{2}$ in 6 -well plates, treated with vehicle/aKG. This procedure was repeated until MSCs reached 
their maximal life span, indicated by the growth arrest when the cells unable to become confluent within 4 weeks of culturing. Cumulative Population Doubling $(\mathrm{CPD})$ was according to the formula $\mathrm{PD}=\log 2(\mathrm{Nh} / \mathrm{Ni}), \mathrm{CPD}=$ sum, $I=1 \ldots \mathrm{nPD}$, where $\mathrm{Nh}=$ harvested number of cells, $\mathrm{Ni}=$ initial number of cells, and $n=$ number of passage.

Senescence-associated- $\beta$-galactosidase assay. The SA- $\beta$-gal activity was detected using the in situ $\beta$-galactosidase staining kit (\#RG0039, Beyotime) following the manufacturer's instructions. MSCs isolated from aged mice (18-20 months old) were seeded into 6-well culture plates and treated with or without aKG for 3 days. The cells were washed twice with PBS and fixed with the $4 \%$ paraformaldehyde for $15 \mathrm{~min}$. After incubation with the SA- $\beta$-gal detection solution at $37^{\circ} \mathrm{C}$ for $2 \mathrm{~h}$, the cells were washed and analyzed under the microscope (Leica) on a scale of $400 \times$ for ten random sites. To evaluate the effect of aKG on $\mathrm{H}_{2} \mathrm{O}_{2}$-induced senescence, cells were treated with $100 \mu \mathrm{M} \mathrm{H} \mathrm{H}_{2} \mathrm{O}_{2}$ for $2 \mathrm{~h}$ and maintained in $10 \mu \mathrm{M} \mathrm{H}_{2} \mathrm{O}_{2}$ for an additional $48 \mathrm{~h}$ in the absence or presence of aKG.

Immunofluorescence staining. Cells were grown on glass slides in 24-well plates. Upon harvest, cells were fixed with $4 \%$ paraformaldehyde and at room temperature (RT) for $20 \mathrm{~min}$. Then, cells were permeabilized with $0.1 \%$ Triton X-100 in PBS at $4{ }^{\circ} \mathrm{C}$ for $10 \mathrm{~min}$. Cells were incubated with $4 \%$ BSA in PBS for $30 \mathrm{~min}$ at $37^{\circ} \mathrm{C}$ to block nonspecific interaction. Primary antibodies were added into the wells and cells were incubated at $4{ }^{\circ} \mathrm{C}$ overnight. Primary antibodies used in this study were as follows: polyclonal anti- $\gamma \mathrm{H} 2 \mathrm{~A} . \mathrm{X}$ (\#9718S, CST, 1:200), polyclonal anti-Lamin A/ C (\#4777S, CST, 1:200), polyclonal anti-H3K9me3 (\#ab8898, Abcam, 1:500), polyclonal anti-H3K27me3 (\#9733S, CST, 1:1000). Next day, primary antibodies were removed, and cells were rinsed with PBS for $5 \mathrm{~min}$. Then we incubated cells at room temperature for $1 \mathrm{~h}$ with the corresponding secondary antibody and washed with PBS for three times afterward. Cells were mounted using an Antifade Mounting Medium with DAPI (\#H-1200, VECTOR).

For immunofluorescence double staining, freshly dissected bone tissues were fixed in $4 \%$ paraformaldehyde for 2 days and decalcified in 10\% EDTA for 2 weeks. Slides were incubated at $99^{\circ} \mathrm{C}$ for $20 \mathrm{~min}$ for antigen retrieval using sodium citrate buffer. Then, sections were incubated with the combination of polyclonal goat antiLepR (\#AF497, R\&D, 1:200) and polyclonal anti-H3K9me3 (\#ab8898, Abcam, 1:500), polyclonal anti-H3K27me3 (\#9733S, CST, 1:1000) at $4{ }^{\circ} \mathrm{C}$ overnight. After washing with PBS solution for three times, the slides were incubated with secondary antibodies of donkey anti-goat FITC (\#bs-0294D-FITC, Bioss, 1:500) and donkey anti-rabbit Alexa Fluor 555 (\#A0453, Beyotime, 1:500) in dark for $1 \mathrm{~h}$ at room temperature. Images of these slides were acquired with a microscope (IX81, Olympus), and quantitative analyses were conducted via ImageJ software.

Quantitative RT-PCR. Total RNA was extracted from cultured MSCs using Trizol (Invitrogen) according to the manufacturer's instruction, and NanoDrop 2000 (Thermo Fisher Scientific) was used to quantify the purity and concentration of RNA. One microgram cDNA of each sample was obtained using the PrimeScript RT reagent Kit with gDNA Eraser (Takara). The real-time PCR was performed using SYBR Premix Ex Taq II (Takara) in CFX96 Real-Time System (Bio-Rad) following the manufacturer's instruction, and the relative expression of target gene was normalized by housekeeping gene (Gapdh) using a $2^{-\Delta \Delta \mathrm{Ct}}$ method subsequently. The primers used for qPCR are presented in Supplementary Table 1.

Western blot. Proteins were isolated in RIPA buffer (Pierce, Rockford, IL) on ice. Lysates were separated by electrophoresis on $12 \%$ SDS-PAGE polyacrylamide gels. Then, samples were electro-transferred to PVDF membranes ( $0.22 \mu \mathrm{m}$, Millipore) using a wet transfer method and blocked with $5 \%$ BSA for $1 \mathrm{~h}$ at room temperature. Then, membranes were incubated overnight at $4{ }^{\circ} \mathrm{C}$ with primary antibodies of $\mathrm{H} 3$ (\#4499, CST, 1:2000), H3K9me3 (\#ab8898, Abcam, 1:1000) and H3K27me3 (\#9733S, CST, 1:1000), H3K4me3 (\#9751, CST, 1:1000), H3K9ac (\#9649, CST, 1:1000), H3K27ac (\#8173, CST, 1:1000), respectively. The next day, blots were incubated with horseradish peroxidase (HRP)-conjugated secondary antibodies (Jackson Immuno Research, West Grove, PA) at room temperature for $1 \mathrm{~h}$. Finally, ECL reagent (Millipore) was used for the visualization and detection of antibodyantigen complexes. The uncropped gel images are shown in Supplementary Fig. 8.

RNA sequencing and GSEA. Total RNAs from aged MSCs with or without $\alpha \mathrm{KG}$ treatment were extracted by Trizol reagent and purified using poly-T oligoattached magnetic beads (Cat. 61006, Thermo). We used NEBNext ${ }^{\oplus}$ Ultra $^{\text {mt }}$ RNA Library Prep Kit for Illumina ${ }^{\circledR}$ (NEB, USA) following the manufacturer's recommendations to generate sequencing libraries ${ }^{74}$. Samples were then subjected to Illumina HiSeq 3000. FastQC (v0.11.5) and FASTX toolkit (0.0.13) were used to control the quality of RNA-seq data, which were then mapped to Mus musculus reference genomes (NCBI build 37, Jul 2007, mm9) using HISAT2 (v.2.0.4). Ballgown software (v.3.4.0) was performed to identify differentially expressed genes and transcripts. Genes were considered significantly differentially expressed if showing $\geq 1.5$ fold change and $P$ value $<0.05$.

For GSEA, the gene sets for osteogenesis and BMP pathway were obtained from Qiagen or GSEA online database, respectively. We imported our gene list of interest into the GSEA software (http://www.broad.mit.edu/GSEA, v.4.0.2) and examined whether this given gene set showed statistically significant. $P$ values were computed using a bootstrap distribution created by resampling gene sets of the same cardinality.

ChIP assay. The enzymatic shearing of chromatin from MSCs was performed using the EZ-Zyme ${ }^{\text {my }}$ Chromatin Prep Kit (\#17-375, Millipore) according to the manufacturer's instructions. Briefly, cells were incubated in $1 \%$ formaldehyde for $10 \mathrm{~min}$ at room temperature to crosslink chromatin, and then stopped by the glycine. Then, the samples were collected and enzymatic sheared step by step with the mixed buffers supplied in this kit. Chromatin was fragmented to $200-400 \mathrm{bp}$, as confirmed by the results of agarose gel electrophoresis. Subsequently, enzymatic sheared chromatins were CHIP-ed using the EZ-Magna ChIP ${ }^{\mathrm{m} x}$ HiSens Chromatin Immunoprecipitation Kit (\#10-10461, Millipore) according to the manufacturer's protocol. The antibodies for ChIP assay were H3K9me3 (\#ab8898, Abcam, $4 \mu \mathrm{g} /$ test), H3K27me3 (\#9733S, CST, $4 \mu \mathrm{g} / \mathrm{test}$ ) and IgG (supplied in the kit, $4 \mu \mathrm{g} / \mathrm{test}$ ). Precipitated DNA samples were quantified with real-time PCR and data are presented as the percentage of input DNA.

Statistics and reproducibility. All values were expressed as mean \pm SEM. Statistically significant differences were assessed by unpaired two-tailed Student's $t$ test for comparison between two groups, one-way ANOVA followed by Tukey's post hoc test and two-way ANOVA followed by Sidak's multiple comparisons tes for multiple comparisons. A $P$ value $<0.05$ was considered statistically significant. Results are from three independent experiments.

Reporting summary. Further information on research design is available in the Nature Research Reporting Summary linked to this article.

\section{Data availability}

The RNA-seq datasets have been submitted to the NCBI database under the accession number GSE139496. Other databases used in the study are Mus musculus reference genomes (NCBI build 37, Jul 2007, mm9), Qiagen (https://www.qiagen.com) and GSEA online database (https://www.gsea-msigdb.org/gsea/index.jsp). The authors declare that all other data supporting the findings of this study are available within the article and its Supplementary information files. Source data are provided with this paper.

Received: 19 May 2019; Accepted: 5 October 2020; Published online: 05 November 2020

\section{References}

1. Johnson, F. B., Sinclair, D. A. \& Guarente, L. Molecular biology of aging. Cell 96, 291-302 (1999).

2. NIH Consensus Development Panel on Osteoporosis Prevention DD, and Therapy. Osteoporosis prevention, diagnosis, and therapy. JAMA 285, 785-795 (2001)

3. Rachner, T. D., Khosla, S. \& Hofbauer, L. C. Osteoporosis: now and the future Lancet 377, 1276-1287 (2011)

4. Saraswat, K. \& Rizvi, S. I. Novel strategies for anti-aging drug discovery. Expert Opin. Drug Discov. 12, 955-966 (2017).

5. Maraka, S. \& Kennel, K. A. Bisphosphonates for the prevention and treatment of osteoporosis. BMJ 351, h3783 (2015).

6. Barrett-Connor, E. \& Stuenkel, C. A. Hormone replacement therapy (HRT)-risks and benefits. Int. J. Epidemiol. 30, 423-426 (2001).

7. Whitaker, M., Guo, J., Kehoe, T. \& Benson, G. Bisphosphonates for osteoporosis-where do we go from here? N. Engl. J. Med. 366, 2048-2051 (2012).

8. Markham, A. Romosozumab: first global approval. Drugs 79, 471-476 (2019)

9. Sleeman, A. \& Clements, J. N. Abaloparatide: a new pharmacological option for osteoporosis. Am. J. Health Syst. Pharm. 76, 130-135 (2019).

10. Lotinun, S. et al. Osteoclast-specific cathepsin K deletion stimulates S1Pdependent bone formation. J. Clin. Invest. 123, 666-681 (2013).

11. Xu, R. Semaphorin 3A: a new player in bone remodeling. Cell Adhes. Migr. 8 5-10 (2014)

12. Arthur, A. et al. EphB4 enhances the process of endochondral ossification and inhibits remodeling during bone fracture repair. J. Bone Miner. Res. 28, 926-935 (2013).

13. Awasthi, H., Mani, D., Singh, D. \& Gupta, A. The underlying pathophysiology and therapeutic approaches for osteoporosis. Med. Res. Rev. 38, 2024-2057 (2018).

14. Zdzisińska, B., Żurek, A. \& Kandefer-Szerszeń, M. Alpha-Ketoglutarate as a molecule with pleiotropic activity: well-known and novel possibilities of therapeutic use. Arch. Immunol. Ther. Exp. 65, 21-36 (2016).

15. Xiao, D. et al. The glutamine-alpha-ketoglutarate (AKG) metabolism and its nutritional implications. Amino Acids 48, 2067-2080 (2016). 
16. Dobrowolski, P., Tomaszewska, E., Bienko, M., Radzki, R. P. \& Pierzynowski, S. G. The effect of dietary administration of 2-oxoglutaric acid on the cartilage and bone of growing rats. Br. J. Nutr. 110, 651-658 (2013).

17. Harrison, A. P., Tygesen, M. P., Sawa-Wojtanowicz, B., Husted, S. \& Tatara, M. R. Alpha-ketoglutarate treatment early in postnatal life improves bone density in lambs at slaughter. Bone 35, 204-209 (2004).

18. Dobrowolski, P. et al. Can 2-oxoglutarate prevent changes in bone evoked by omeprazole? Nutrition 29, 556-561 (2013).

19. Sliwa, E. 2-Oxoglutaric acid administration diminishes fundectomy-induced osteopenia in pigs. J. Anim. Physiol. Nutr. 94, e86-e95 (2010).

20. Radzki, R. P., Bienko, M., Filip, R. \& Pierzynowski, S. G. The protective and therapeutic effect of exclusive and combined treatment with Alphaketoglutarate sodium salt and ipriflavone on bone loss in orchidectomized rats. J. Nutr. Health Aging 20, 628-636 (2016).

21. Carey, B. W., Finley, L. W., Cross, J. R., Allis, C. D. \& Thompson, C. B. Intracellular alpha-ketoglutarate maintains the pluripotency of embryonic stem cells. Nature 518, 413-416 (2015).

22. Chin, R. M. et al. The metabolite alpha-ketoglutarate extends lifespan by inhibiting ATP synthase and TOR. Nature 510, 397-401 (2014).

23. Glatt, V., Canalis, E., Stadmeyer, L. \& Bouxsein, M. L. Age-related changes in trabecular architecture differ in female and male C57BL/6J mice. J. Bone Miner. Res 22, 1197-1207 (2007).

24. Halloran, B. P. et al. Changes in bone structure and mass with advancing age in the male C57BL/6J mouse. J. Bone Miner. Res. 17, 1044-1050 (2002).

25. Liu, W. et al. GDF11 decreases bone mass by stimulating osteoclastogenesis and inhibiting osteoblast differentiation. Nat. Commun. 7, 12794 (2016).

26. Bellantuono, I., Aldahmash, A. \& Kassem, M. Aging of marrow stromal (skeletal) stem cells and their contribution to age-related bone loss. Biochim. Biophys. Acta 1792, 364-370 (2009).

27. Sethe, S., Scutt, A. \& Stolzing, A. Aging of mesenchymal stem cells. Ageing Res. Rev. 5, 91-116 (2006).

28. Scaffidi, P. \& Misteli, T. Lamin A-dependent misregulation of adult stem cells associated with accelerated ageing. Nat. Cell Biol. 10, 452-459 (2008).

29. Mah, L. J., El-Osta, A. \& Karagiannis, T. C. $\gamma \mathrm{H} 2 \mathrm{AX}$ : a sensitive molecular marker of DNA damage and repair. Leukemia 24, 679-686 (2010).

30. $\mathrm{Wu}, \mathrm{H}$. et al. Chromatin dynamics regulate mesenchymal stem cell lineage specification and differentiation to osteogenesis. Biochim. Biophys. Acta 1860, 438-449 (2017).

31. Hemming, S. et al. EZH2 and KDM6A act as an epigenetic switch to regulate mesenchymal stem cell lineage specification. Stem Cells 32, 802-815 (2014).

32. Ye, L. et al. Histone demethylases KDM4B and KDM6B promotes osteogenic differentiation of human MSCs. Cell Stem Cell 11, 50-61 (2012).

33. Zhou, B. O., Yue, R., Murphy, M. M., Peyer, J. G. \& Morrison, S. J. Leptinreceptor-expressing mesenchymal stromal cells represent the main source of bone formed by adult bone marrow. Cell Stem Cell 15, 154-168 (2014).

34. Zhang, D. et al. LepR-expressing stem cells are essential for alveolar bone regeneration. J. Dent. Res. 99, 1279-1286 (2020).

35. Silva, J. et al. Nanog is the gateway to the pluripotent ground state. Cell 138, 722-737 (2009).

36. Wang, L. et al. A small molecule modulates Jumonji histone demethylase activity and selectively inhibits cancer growth. Nat. Commun. 4, 2035 (2013).

37. Conboy, I. M., Conboy, M. J. \& Rebo, J. Systemic problems: a perspective on stem cell aging and rejuvenation. Aging 7, 754-765 (2015).

38. TeSlaa, T. et al. alpha-Ketoglutarate accelerates the initial differentiation of primed human pluripotent stem cells. Cell Metab. 24, 485-493 (2016).

39. Lu, V. \& Teitell, M. A. Alpha-ketoglutarate: a "magic" metabolite in early germ cell development. Embo J. 38, e100615 (2019).

40. Lin, A. L., Zhang, W., Gao, X. \& Watts, L. Caloric restriction increases ketone bodies metabolism and preserves blood flow in aging brain. Neurobiol. Aging 36, 2296-2303 (2015).

41. Niemiec, T. et al. Alpha-ketoglutarate stabilizes redox homeostasis and improves arterial elasticity in aged mice. J. Physiol. Pharm. 62, 37-43 (2011).

42. Blonde-Cynober, F., Aussel, C. \& Cynober, L. Use of ornithine alphaketoglutarate in clinical nutrition of elderly patients. Nutrition 19, 73-75 (2003).

43. Coudray-Lucas, C., Le Bever, H., Cynober, L., De Bandt, J. P. \& Carsin, H. Ornithine alpha-ketoglutarate improves wound healing in severe burn patients: a prospective randomized double-blind trial versus isonitrogenous controls. Crit. Care Med. 28, 1772-1776 (2000).

44. Harrison, A. P. \& Pierzynowski, S. G. Biological effects of 2-oxoglutarate with particular emphasis on the regulation of protein, mineral and lipid absorption/ metabolism, muscle performance, kidney function, bone formation and cancerogenesis, all viewed from a healthy ageing perspective state of the artreview article. J. Physiol. Pharm. 59, 91-106 (2008).

45. Tian, Q. et al. Dietary alpha-ketoglutarate promotes beige adipogenesis and prevents obesity in middle-aged mice. Aging Cell 19, e13059 (2020).

46. Almeida, M. et al. Skeletal involution by age-associated oxidative stress and its acceleration by loss of sex steroids. J. Biol. Chem. 282, 27285-27297 (2007).
47. Jilka, R. L. The relevance of mouse models for investigating age-related bone loss in humans. J. Gerontol. Ser. A 68, 1209-1217 (2013).

48. Sankaran, J. S., Varshney, M. \& Judex, S. Differences in bone structure and unloading-induced bone loss between C57BL/6N and C57BL/6J mice. Mamm. Genome 28, 476-486 (2017).

49. Maddatu, T. P., Grubb, S. C., Bult, C. J. \& Bogue, M. A. Mouse phenome database (MPD). Nucleic Acids Res. 40, D887-D894 (2012).

50. Willinghamm, M. D. et al. Age-related changes in bone structure and strength in female and male BALB/c mice. Calcif. Tissue Int. 86, 470-483 (2010).

51. Manolagas, S. C. From estrogen-centric to aging and oxidative stress: a revised perspective of the pathogenesis of osteoporosis. Endocr. Rev. 31, 266-300 (2010).

52. Yu, B. \& Wang, C. Y. Osteoporosis: the result of an 'Aged' bone microenvironment. Trends Mol. Med. 22, 641-644 (2016).

53. Li, L. et al. Aging increases the susceptivity of MSCs to reactive oxygen species and impairs their therapeutic potency for myocardial infarction. PLOS ONE 9, e111850 (2014)

54. Liu, S., He, L. \& Yao, K. The antioxidative function of alpha-ketoglutarate and its applications. Biomed. Res. Int. 2018, 6 (2018).

55. Fu, X. et al. 2-Hydroxyglutarate inhibits ATP synthase and mTOR signaling. Cell Metab. 22, 508-515 (2015).

56. Xie, Y. et al. Defining the role of oxygen tension in human neural progenitor fate. Stem Cell Rep. 3, 743-757 (2014)

57. Mathieu, J. et al. Hypoxia-inducible factors have distinct and stage-specific roles during reprogramming of human cells to pluripotency. Cell Stem Cell 14, 592-605 (2014).

58. Zhang, W., Qu, J., Liu, G. H. \& Belmonte, J. C. I. The ageing epigenome and its rejuvenation. Nat. Rev. Mol. Cell Biol. 21, 137-150 (2020).

59. Huidobro, C., Fernandez, A. F. \& Fraga, M. F. Aging epigenetics: causes and consequences. Mol. Asp. Med. 34, 765-781 (2013).

60. Jirtle, R. L. \& Skinner, M. K. Environmental epigenomics and disease susceptibility. Nat. Rev. Genet. 8, 253-262 (2007).

61. Wang, Y., Yuan, Q. \& Xie, L. Histone modifications in aging: the underlying mechanisms and implications. Curr. Stem Cell Res. Ther. 13, 125-135 (2018).

62. Wood, J. G. et al. Chromatin remodeling in the aging genome of Drosophila Aging Cell 9, 971-978 (2010).

63. D’Aquila, P., Rose, G., Bellizzi, D. \& Passarino, G. Epigenetics and aging. Maturitas 74, 130-136 (2013).

64. Xiao, M. et al. Inhibition of alpha-KG-dependent histone and DNA demethylases by fumarate and succinate that are accumulated in mutations of FH and SDH tumor suppressors. Genes Dev. 26, 1326-1338 (2012).

65. Meier, J. L. Metabolic mechanisms of epigenetic regulation. ACS Chem. Biol. 8, 2607-2621 (2013)

66. Martinez, S. \& Hausinger, R. P. Catalytic mechanisms of Fe(II)- and 2oxoglutarate-dependent oxygenases. J. Biol. Chem. 290, 20702-20711 (2015).

67. Alves, J., Vidugiris, G., Goueli, S. A. \& Zegzouti, H. Bioluminescent highthroughput succinate detection method for monitoring the activity of JMJC histone demethylases and $\mathrm{Fe}(\mathrm{II}) / 2$-oxoglutarate-dependent dioxygenases. SLAS Discov. 23, 242-254 (2017).

68. Su, X., Wellen, K. E. \& Rabinowitz, J. D. Metabolic control of methylation and acetylation. Curr. Opin. Chem. Biol. 30, 52-60 (2016).

69. Deng, P., Chen, Q. M., Hong, C. \& Wang, C. Y. Histone methyltransferases and demethylases: regulators in balancing osteogenic and adipogenic differentiation of mesenchymal stem cells. Int. J. Oral. Sci. 7, 197-204 (2015)

70. Li, Q. et al. Ubiquitin-specific protease 34 inhibits osteoclast differentiation by regulating NF-kappaB signaling. J. Bone Miner. Res. 35, 1597-1608 (2020).

71. Guo, Y. C. et al. Ubiquitin-specific protease USP34 controls osteogenic differentiation and bone formation by regulating BMP2 signaling. Embo J. 37, e99398 (2018).

72. Soleimani, M. \& Nadri, S. A protocol for isolation and culture of mesenchymal stem cells from mouse bone marrow. Nat. Protoc. 4, 102-106 (2009).

73. Zhao, X. et al. ZBP1 (DAI/DLM-1) promotes osteogenic differentiation while inhibiting adipogenic differentiation in mesenchymal stem cells through a positive feedback loop of Wnt/beta-catenin signaling. Bone Res. 8, 12 (2020).

74. Wu, Y. et al. Mettl3-mediated m(6)A RNA methylation regulates the fate of bone marrow mesenchymal stem cells and osteoporosis. Nat. Commun. 9, 4772 (2018)

\section{Acknowledgements}

We thank Qiang Guo from the State Key Laboratory of Oral Diseases for providing instructions on microCT analyses. We thank Ping Yi and Linlin Zhang from West China Hospital of Stomatology, Yanqiu Gong and Lunzhi Dai from the State Key Laboratory of Biotherapy, Sichuan University for the assistance of aKG measurement. This work was supported by grants from the National Natural Science Foundation of China (NSFC 81722014, 81970913), State Key Laboratory of Oral Diseases (SKLOD201804) and National Key R\&D Program of China during the 13th Five-Year Plan (2016YFC1102700). 


\section{Author contributions}

Y.W., P.D., C.W., and Q.Y. designed the project; Y.W., P.D., Y.L., X.Z., Y.Wu, S.Z., and Y.G. performed the in vitro experiments; Y.W., P.D., X.Z., Y.L., L.Z., Y.G., Y.Wu, W.L., Q.L., X.Q., and Y.C. performed the in vivo experiments; Y.W., P.D., Q.L., W.L., G.O., and Q.Y. analyzed the data; Y.W., P.D., C.W., and Q.Y. wrote and edited the manuscript. All authors reviewed the manuscript.

\section{Competing interests}

The authors declare no competing interests.

\section{Additional information}

Supplementary information is available for this paper at https://doi.org/10.1038/s41467020-19360-1.

Correspondence and requests for materials should be addressed to Q.Y.

Peer review information Nature Communications thanks Jesus Delgado-Calle, Stefan Pierzynowski and the other, anonymous, reviewer(s) for their contribution to the peer review of this work.
Reprints and permission information is available at http://www.nature.com/reprints

Publisher's note Springer Nature remains neutral with regard to jurisdictional claims in published maps and institutional affiliations.

(c) (i) Open Access This article is licensed under a Creative Commons Attribution 4.0 International License, which permits use, sharing, adaptation, distribution and reproduction in any medium or format, as long as you give appropriate credit to the original author(s) and the source, provide a link to the Creative Commons license, and indicate if changes were made. The images or other third party material in this article are included in the article's Creative Commons license, unless indicated otherwise in a credit line to the material. If material is not included in the article's Creative Commons license and your intended use is not permitted by statutory regulation or exceeds the permitted use, you will need to obtain permission directly from the copyright holder. To view a copy of this license, visit http://creativecommons.org/ licenses/by/4.0/.

(C) The Author(s) 2020 